\title{
Pacific
}

Journal of

Mathematics

EXPLICIT CONSTRUCTION OF CERTAIN SPLIT EXTENSIONS OF NUMBER FIELDS AND CONSTRUCTING CYCLIC CLASSFIELDS

STANLEy JOSEPH GURAK

Volume $157 \quad$ No. 2

February 1993 


\section{EXPLICIT CONSTRUCTION OF \\ CERTAIN SPLIT EXTENSIONS OF NUMBER FIELDS AND CONSTRUCTING CYCLIC CLASSFIELDS}

\section{S. GURAK}

The problem of explicitly constructing classfields (Hilbert's Twelfth problem) is largely unresolved, except when a classfield is absolutely abelian or abelian over an imaginary quadratic number field. Here an explicit construction of certain split extensions of number fields is given, which maintains control over the primes which ramify. This naturally leads to the construction of cyclic classifields over a given number field. An algorithm is provided to obtain the minimal polynomials for the generating elements of the extension constructed. The methods employed here rely heavily on classfield theory and the properties of Lagrange resolvents and group determinants.

1. Introduction. The principal goal of this research is to obtain an explicit construction of certain split algebraic extension fields over a given number field $F$. The characterization of such fields is given for dihedral extensions of degree 6,8 and 12 over $Q$ in a previous work [6]. Those constructions rely on the arithmetic of quadratic fields and explicit formulas such as Cardano's. The constructions given here rely on classfield theory and the properties of Lagrange resolvents. There is a natural extension of the construction for similar extensions over function fields which will be treated in a subsequent paper.

To be more precise about the extensions we seek to construct, let $Z_{n}$ denote the ring of residues modulo $n$, for some integer $n>1$, with unit group $Z_{n}^{*}$. Consider a polynomial $p(x)=x^{n}+a_{1} x^{n-1}+\cdots+a_{0}$, irreducible over $F$, with Galois group $G$ of the form $V \cdot T$, where $T$ is cyclic of order $n$ with $T \unlhd G$ and $V$ is isomorphic to a subgroup of $Z_{n}^{*}$ (that is, $G$ is a semi-direct product of $T$ by $V$ ). Let $K$ be the splitting field of $p(x)$ so $G(K / F)=G$, and let $k$ and $R$ be the subfields fixed by $T$ and $V$ respectively. We wish to give an explicit general construction for the extension $K / F$ (or $K / k)$ in terms of the arithmetic of $k(\zeta)$, where $\zeta=\exp (2 \pi i / n)$. Since $K=k \cdot R$, the problem of determining $K$ primarily is one of finding explicit generators for the field $R$ (or a conjugate field). Of course, $R$ is generated by some root of $p(x)$, but actually finding roots of $p(x)$ 
in terms of radicals is generally quite tedious. The strategy here is to replace $p(x)$ by a collection of irreducible polynomials of prime power degrees, each of whose roots are explicitly found in terms of radicals. The collection is so chosen that $R$ is generated by a certain choice of roots, one from each polynomial in the collection.

To carry out this approach, first note that the multiplication in $G$ is explicitly given in terms of a generator $\sigma$ of $T$ via

$$
\tau \sigma \tau^{-1}=\sigma^{\theta(\tau)} \quad(\tau \in V),
$$

for some homomorphism $\theta: V \rightarrow Z_{n}^{*}$. (The map $\theta$ is independent of the choice of generator $\sigma$, and in fact, a monomorphism since $K$ is the splitting field of $R$.) Write $n=n_{1} n_{2} \cdots n_{t}$ as a product of distinct prime powers, and let $\pi_{i}$ denote the natural projection of $Z_{n}^{*}$ onto $Z_{n_{i}}^{*}(1 \leq i \leq t)$. Set $\theta_{i}=\pi_{i} \circ \theta$ with

$$
\operatorname{ker} \theta_{i}=\left\{\tau \in V \mid \theta(\tau) \equiv 1\left(\bmod n_{i}\right)\right\} \quad(1 \leq i \leq t) .
$$

and put $T_{i}=\left\langle\sigma^{n_{i}}\right\rangle$ for $1 \leq i \leq t$. The subgroups $\left(\operatorname{ker} \theta_{i}\right) \quad T_{i} \quad(1 \leq$ $i \leq t$ ) are easily seen to be normal in $G$. Let $K_{i}, R_{i}$ and $k_{i}$ be the subfields of $K$ fixed by $\left(\operatorname{ker} \theta_{i}\right) T_{i}, V \cdot T_{i}$ and $\left(\operatorname{ker} \theta_{i}\right) T$ respectively $(1 \leq i \leq t)$. Then one has the following correspondence from Galois theory
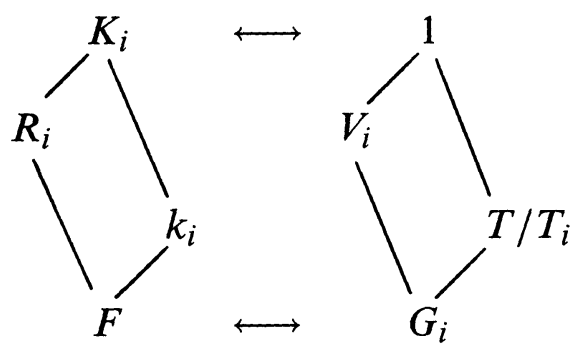

where $V_{i}=V /\left(\operatorname{ker} \theta_{i}\right)$ and $G_{i}=V_{i} \cdot T /\left(T_{i}\right) \quad(1 \leq i \leq t)$. Since $\bigcap \operatorname{ker} \theta_{i}=1$, and $\cap T_{i}=1$, we find that $K=\prod K_{i}, R=\prod R_{i}$ and $k=\prod k_{i}$. In particular, $\operatorname{deg}\left(R_{i} / F\right)=n_{i}$ and $\operatorname{deg}\left(k_{i} / F\right)=$ $\left[V: \operatorname{ker} \theta_{i}\right]$. We have shown

Proposition 1. To construct the splitting field $K$, it suffices to find generating elements $\alpha_{i}$ for the fields $R_{i}$ and $\gamma_{i}$ for the abelian extensions $k_{i}$ over $F$.

We note that the subfields $k_{i}$ can be exhibited explicitly from the arithmetic of $k$ in a straightforward manner using Galois theory (for instance, by taking appropriate norms or traces). Thus the above result 
reduces the construction of $R$ to that of finding explicit generators for each of the subfields $R_{i}$ of prime power degree over $F$. Our strategy to construct such a given subfield is to exhibit a particular generator, which is explicitly given in terms of radicals over a suitable resolvent field, together with its minimal polynomial of prime power degree. A general algorithm for this construction is given in $\S 3$ with explicit examples appearing later in $\S 4$.

2. Characterization of the resolvent field. Recall the situation just described with $n=n_{1} \cdots n_{t}$ as a product of distinct prime powers. The extension $K$ is the splitting field of some irreducible polynomial of degree $n$ over $F$ whose Galois group $G=V \cdot T$ is a semi-direct product of a cyclic group $T$ of order $n$ by a group $V$ isomorphic to a subgroup of $Z_{n}^{*}$. The subfields $k$ and $R$ are those fixed by $T$ and $V$ respectively in the Galois correspondence. The mapping $\theta: V \rightarrow Z_{n}^{*}$ in (1) can be determined from classfield theory, without explicitly determining $K$, using only arithmetic in the abelian extension $k / F$. Indeed, $K / k$ is classfield to a certain ideal group $\mathbf{J}$ defined for a suitable $k$-modulus $\mathscr{M}$. So if $\mathbf{I}$ is the group of fractional $k$-ideals prime to $\mathscr{M}$, the quotient $\mathbf{I} / \mathbf{J} \cong G(K / k)=T$ through Artin reciprocity. We shall indicate the Artin map of $K / k$ by

$$
[\sigma] \rightarrow \sigma \text { for } \sigma \text { in } T \text {, }
$$

where $[\sigma]$ represents the coset of $\mathbf{I} / \mathbf{J}$ which maps to $\sigma$ in $T$. Since $T \unlhd G$ we have $\tau(\mathbf{J})=\mathbf{J}$, so $\tau([\sigma])=\left[\tau \sigma \tau^{-1}\right]=\left[\sigma^{\theta(\tau)}\right]$ from properties of the Artin map. To actually determine $\theta$ one needs only to find a prime ideal $\rho$ in the coset $[\sigma]$, then compute the coset to which $\tau(\mu)$ belongs for each $\tau$ in $V$. For a given generator $\alpha$ for $R / F$ of zero trace and fixed generator $\sigma$ of $T$, form the Lagrange resolvents

$$
\omega_{\nu}=\omega_{\nu}(\alpha)=\alpha+\zeta^{-\nu} \sigma(\alpha)+\cdots+\zeta^{-(n-1) \nu} \sigma^{n-1}(\alpha) \quad\left(\nu \in Z_{n}\right) .
$$

Set

$$
\alpha_{\mu}=\frac{1}{n} \sum_{\nu=1}^{n-1} \zeta^{\mu \nu} \omega_{\nu} \quad\left(\mu \in Z_{n}\right)
$$

where $\zeta=\zeta_{n}=\exp (2 \pi i / n)$, so that for $0 \leq \lambda<n$,

$$
\sigma^{\lambda}\left(\alpha_{\mu}\right)=\alpha_{\mu+\lambda} \quad\left(\mu \in Z_{n}\right) .
$$

The elements $\alpha_{\mu}$ is (6) are the distinct zeros of an irreducible polynomial

$$
p(x)=x^{n}+a_{1} x^{n-1}+\cdots+a_{n}
$$


of degree $n$, with $a_{1}=0$, whose splitting field is $K$. By replacing $\alpha$ by a suitable conjugate, we may assume that $\omega_{1} \neq 0$.

It is known that the elements $\beta_{\nu}=\omega_{\nu}^{n}\left(\nu \in Z_{n}\right)$ lie in $k(\zeta)$. (This follows readily from the proof of Lemma 1 below.) The $\beta_{\nu}$ actually lie in some subfield $k^{\prime}$ called the resolvent field for $p(x)$ over $F$, which can be described in terms of Galois theory in the following manner.

Label each element $\phi$ of $G(k(\zeta) / F)$ by $\phi=\phi_{\tau, s}$, where $\tau$ is the unique map in $V$ which coincides with $\phi$ on $k$, and $s$ in $Z_{n}^{*}$ is determined by the action $\phi(\zeta)=\zeta^{s}$. The resolvent field $k^{\prime}$ is that which is fixed by the subgroup $W=\left\{\phi_{\tau, s} \mid s \equiv \theta(\tau) \bmod n\right\}$. Since $\phi_{1,1}$ is the only map in $W$ that fixes $k$, one finds that $k^{\prime}(\zeta)=k(\zeta)=$ $k^{\prime} k$. That $k^{\prime}$ contains the $\beta_{\nu} \quad(0 \leq \nu<n)$ is immediate from the next lemma.

LEMMA 1. For each $\phi=\phi_{\tau, s}$ in $G(k(\zeta) / F)$ and integer $\nu, \phi_{\tau, s}\left(\beta_{\nu}\right)$ $=\beta_{\nu s \theta\left(\tau^{-1}\right)}$.

Proof. Let $\tilde{\phi}$ be any extension of $\phi$ to $K(\zeta)$, say with $\left.\tilde{\phi}\right|_{K}=\tau \sigma^{t}$ in $G$ for some integer $t, 0 \leq t<n$. First note that from (1) and (7),

$$
\tilde{\phi}\left(\alpha_{\mu}\right)=\tau \sigma^{t}\left(\alpha_{\mu}\right)=\tau \sigma^{t+\mu}(\alpha)=\sigma^{(t+\mu) \theta(\tau)} \tau(\alpha)=\alpha_{(t+\mu) \theta(\tau)} .
$$

Thus, from (4), we have

$$
\begin{aligned}
\tilde{\phi}\left(\omega_{\nu}\right) & =\sum_{\mu \in Z_{n}} \zeta^{-\mu \nu s} \alpha_{(t+\mu) \theta(\tau)}=\sum_{\mu \in Z_{n}} \zeta^{-\nu s\left(\mu \theta\left(\tau^{-1}\right)-t\right)} \alpha_{\mu} \\
& =\zeta^{t s \nu} \sum_{\mu \in Z_{n}} \zeta^{-\nu s \mu \theta\left(\tau^{-1}\right)} \alpha_{\mu}=\zeta^{t s \nu} \omega_{s \theta\left(\tau^{-1}\right) \nu}
\end{aligned}
$$

upon replacing $\mu$ by $\mu \theta\left(\tau^{-1}\right)-t$ in the first summation. In particular, $\phi\left(\beta_{\nu}\right)=\beta_{s \theta\left(\tau^{-1}\right) \nu}$.

The resolvent field $k^{\prime}$ has a convenient characterization in terms of class field theory. Suppose $k / F$ has conductor $\ell$ and put $m=$ $\operatorname{LCM}\left(n O_{F}, f\right)$, where $O_{F}$ is the ring of integers of $F$. Let $\mathscr{C}$ denote the group of fractional $F$-ideals prime to $m$ and $\mathscr{A}$ be the subgroup of $\mathscr{C}$ which corresponds to the classfield $k$ over $F$. The Artin map of $k / F$ induces an isomorphism of $\mathscr{C} / \mathscr{A}$ with $V$, which we shall indicate by

$$
[\tau] \rightarrow \tau .
$$


(Here [ $\tau$ ] represents the coset of $\mathscr{C} / \mathscr{A}$ which maps to $\left.\tau\right|_{k}$ in $G(k / F)$ via the Artin map.) In a similar way, let $[\phi]$ represent the coset of fractional $F$-ideals in $\mathscr{C}$ which are sent to $\phi$ in $G(k(\zeta) / F)$ by the Artin map of $k(\zeta) / F$. In addition, let $\mathscr{N}=\mathscr{N}_{F / Q}$ denote the absolute ideal norm mapping from $F$ to $Q$. It follows from the properties of the Artin map that for $\phi=\phi_{\tau, s}$ in $G(k(\zeta) / F)$,

$$
\left[\phi_{\tau, s}\right]=\left\{a \in[\tau] \mid N_{a} \equiv s(\bmod n)\right\} \text {. }
$$

The resolvent field $k^{\prime} / F$ corresponds through classfield theory to the group

$$
\mathscr{B}=\bigcup_{\phi \in W}[\phi] .
$$

To aid in the computation of $k^{\prime}$, we mention the following result.

Proposition 1. Let $h(x)$ be any irreducible polynomial of degree $[k: F]$ over $F$ with splitting field $k$. Select a zero $\lambda$ of $h(x)$ and label its conjugates

$$
\tau(\lambda)=\lambda_{\theta(\tau)} \quad(\tau \in V) .
$$

Then the elements $E_{\mu}=\sum_{\tau \in V} \zeta^{-\mu \theta(\tau)} \lambda_{\theta(\tau)}(0 \leq \mu<n)$ all lie in the resolvent field $k^{\prime}$.

Proof. Since $E_{\mu}=k(\zeta)$, it suffices to show that each map in $W$ fixes $E_{\mu}$. But, for any $\phi=\phi_{\tau^{\prime}, \theta\left(\tau^{\prime}\right)}$ in $W$ and $0 \leq \mu<n$,

$$
\phi\left(E_{\mu}\right)=\sum_{\tau \in V} \zeta^{-\mu \theta(\tau) \theta\left(\tau^{\prime}\right)} \lambda_{\theta(\tau) \theta\left(\tau^{\prime}\right)}=E_{\mu} \quad \text { from (12). }
$$

If $K \cap Q(\zeta)=Q$ then

$$
\begin{aligned}
& G(k(\zeta) / F)=\left\{\phi_{\tau, s} \mid \tau \in V, s \in Z_{n}^{*}\right\} \quad \text { and } \\
& G(k(\zeta) / k)=\left\{\phi_{1, s} \mid s \in Z_{n}^{*}\right\} .
\end{aligned}
$$

In particular, we have

Proposition 2. Suppose $K \cap Q(\zeta)=Q$. Then $G(k(\zeta) / k) \cong G\left(k^{\prime} / F\right)$ and $k \cap k^{\prime}=F$.

Proof. The action $\phi_{1, s} \rightarrow \phi_{1, s} W\left(s \in Z_{n}^{*}\right)$ yields a monomorphism of $G(k(\zeta) / k)$ into $G\left(k^{\prime} / F\right)$ since $k^{\prime} k=k(\zeta)$. Also $\phi_{\tau, s}=\phi_{1, s \theta\left(\tau^{-1}\right)^{\circ}}$ $\phi_{\tau, \theta(\tau)}$ here for any $\tau \in V$ and $s \in Z_{n}^{*}$, so the map is clearly surjective. Hence $G(k(\zeta) / k) \cong G\left(k^{\prime} / F\right)$ and $k \cap k^{\prime}=F$. 
EXAMPLE 1. To illustrate the preceding theory with $n=5$ and $F=$ $Q$, consider the complex quartic abelian field $k=Q(\Lambda)$, where $\Lambda=$ $\zeta_{16}+\zeta_{16}^{7}$, of conductor $f=16$ and class number $h=1$ [13]. The ring of integers of $k$ has fundamental unit $E=1+\sqrt{2}$ and integral basis $\{1, \sqrt{2}, \Lambda, \sqrt{2} \Lambda\}$. The field $k$ over $Q$ corresponds through classfield theory to the subgroup $\mathscr{A}=\{(x) \mid x \equiv 1,7,17,23,33,39,49,71$ $(\bmod 80), x>0\}$ defined with $m=80$. It admits an ideal group $J$ defined modulo $\mathscr{M}=\left(5^{2}\right)$ consisting of principal ideals generated by elements $\gamma$ prime to $\mathscr{M}$ and of the form $\gamma=\alpha \cdot \beta$ for some $\alpha \in\left(k^{x}\right)^{5}$ and $\beta \equiv 1+5(\delta+a(1+2 \sqrt{2}) \Lambda) \bmod \mathscr{M}$ with $a \in Z, \delta \in Q(\sqrt{2})$. The ideal group $J$ corresponds to a classfield $K$ which is normal over $Q$ with $[K: k]=5$. The Galois group $G=G(K / Q)$ is a semidirect product of the form $V \cdot T$ as we have been discussing, say with $T=G(K / k)$ generated by an element $\sigma$ for which $[\sigma]=(1+5 \sqrt{2} \Lambda) J$ in (4). Incidentally, we find $\left[\sigma^{j}\right]=(1+5 j \sqrt{2} \Lambda) J(0 \leq j \leq 4)$. Also, if $\tau \in V \cong G(k / Q)$ is given by the action $\Lambda \rightarrow \bar{\Lambda}=\zeta_{16}^{11}+\zeta_{16}^{13}=$ $-(\sqrt{2}+1) \Lambda$, then $\tau[\sigma]=(1-5 \sqrt{2} \Lambda) J=(1+5(2+\sqrt{2}) \Lambda) J=$ $(1+10 \sqrt{2} \Lambda) J$. Thus $\theta(\tau)=2$ in (1). Further, we find $\theta\left(\tau^{2}\right)=4$, $\theta\left(\tau^{3}\right)=3$ and $\theta(1)=1$.

Suppose $\alpha$ is a generator for the field $R$ fixed by $V$, so chosen that the Lagrange resolvents $(5)$ satisfy $\omega_{\nu}=0$ for $(\nu, 5)>1$. To determine the resolvent field $k^{\prime}$ for its minimal polynomial $p(x)$ in (8), we find the corresponding subgroup $\mathscr{B}$ of $\mathscr{C}$ from (11). Noting that

$$
\begin{aligned}
& {[\tau]=\{(x) \mid x>0, x \equiv 11,13,27,29,43,59,61,77(\bmod 80)\},} \\
& {\left[\tau^{2}\right]=\{(x) \mid x>0, x \equiv 9,31,41,47,57,63,73,79(\bmod 80)\}} \\
& \text { and } \\
& {\left[\tau^{3}\right]=\{(x) \mid x>0, x \equiv 3,19,21,37,51,53,67,69(\bmod 80)\}}
\end{aligned}
$$

in (9), we find the sets $[\phi]$ in (11) are

$$
\begin{aligned}
{\left[\phi_{1,1}\right] } & =\{(x) \mid x>0, x \equiv 1,71(\bmod 80)\}, \\
{\left[\phi_{\tau, 2}\right] } & =\{(x) \mid x>0, x \equiv 27,77(\bmod 80)\}, \\
{\left[\phi_{\tau^{2}, 4}\right] } & =\{(x) \mid x>0, x \equiv 9,79(\bmod 80)\}, \quad \text { and } \\
{\left[\phi_{\tau^{3}, 3}\right] } & =\{(x) \mid x>0, x \equiv 3,53(\bmod 80)\} .
\end{aligned}
$$

Thus, we have $\mathscr{B}=\{(x) \mid x>0, x \equiv 1,3,9,27,53,71,77,79$ $(\bmod 80)\}$ with corresponding field $k^{\prime}=Q(\sqrt{10+3 \sqrt{10}})$ from classfield theory. 
Alternatively, we could use Proposition 1 , choosing $\lambda=\Lambda$, to find that

$$
\begin{aligned}
E_{-1}^{2} & =\left[\zeta \Lambda+\zeta^{2} \bar{\Lambda}+\zeta^{3}(-\bar{\Lambda})+\zeta^{4}(-\Lambda)\right]^{2}=\left[\left(\zeta-\zeta^{4}\right) \Lambda+\left(\zeta^{2}-\zeta^{3}\right) \bar{\Lambda}\right]^{2} \\
& =\frac{1}{2}(-5-\sqrt{5})(-2+\sqrt{2})\left[1+\frac{1}{2}(1-\sqrt{5})(1+\sqrt{2})\right]^{2} \\
& =10-3 \sqrt{10}
\end{aligned}
$$

Hence $k^{\prime}=Q(\sqrt{10+3 \sqrt{10}})$.

EXAMPLE 2. Now consider $F=Q(\gamma)$ of narrow class number $h^{+}=$ 1 , where $\gamma=\gamma_{1}$ is the positive zero of $r(x)=x^{3}-x-1$ and $\gamma_{2}$ and $\gamma_{3}$ denote the complex ones. The group $U_{F}^{+}$of totally positive units is seen to be generated by $\gamma$. Choose square roots $\gamma_{1}^{1 / 2}, \gamma_{2}^{1 / 2}, \gamma_{3}^{1 / 2}$ with $\gamma_{2}^{1 / 2} \cdot \gamma_{3}^{1 / 2}=\gamma_{1}^{-1 / 2}>0$. The extension $K=Q\left(\gamma_{1}^{1 / 2}, \gamma_{2}^{1 / 2}, \gamma_{3}^{1 / 2}\right)$ has dihedral Galois group $G=D_{4}$ over $F$ generated by automorphisms $\sigma$ and $\tau$ in (1) satisfying

$$
\begin{array}{llll}
\sigma: & \gamma_{1}^{1 / 2} \rightarrow-\gamma_{1}^{1 / 2}, & \gamma_{2}^{1 / 2} \rightarrow \gamma_{3}^{1 / 2}, & \gamma_{3}^{1 / 2} \rightarrow-\gamma_{2}^{1 / 2} \\
\tau: & \gamma_{1}^{1 / 2} \rightarrow \gamma_{1}^{1 / 2}, & \gamma_{2}^{1 / 2} \rightarrow \gamma_{3}^{1 / 2}, & \gamma_{3}^{1 / 2} \rightarrow \gamma_{2}^{1 / 2} .
\end{array}
$$

The field fixed by $\langle\tau\rangle$ is $R=F\left(\gamma_{1}^{1 / 2}, \gamma_{2}^{1 / 2}+\gamma_{3}^{1 / 2}\right)$ that by $\langle\sigma\rangle$ is $k=F\left(\gamma_{1}^{1 / 2}\left(\gamma_{2}-\gamma_{3}\right)\right)$. The element $\alpha=\gamma_{2}^{1 / 2}+\gamma_{3}^{1 / 2}$ satisfies the minimal polynomial $p(x)=x^{4}+2 \gamma x^{2}+\left(4-3 \gamma^{2}\right)$ in (8) with splitting field $K$ over $F$. Choosing $\lambda=\gamma_{1}^{1 / 2}\left(\gamma_{2}-\gamma_{3}\right)$ in Proposition 1 , we deduce that the resolvent field for $p(x)$ is $k^{\prime}=F\left(i \gamma_{1}^{1 / 2}\left(\gamma_{2}-\gamma_{3}\right)\right)$. Direct computation of $\beta_{1}$ and $\beta_{-1}$ from the Lagrange resolvents $\omega_{1}(\alpha)$ and $\omega_{-1}(\alpha)$ in $(5)$ yields

$$
\beta_{ \pm 1}=64\left(7 \gamma^{2}-8 \pm 4 i\left(\gamma_{2}-\gamma_{3}\right) / \gamma_{1}^{1 / 2}\right) \text {. }
$$

The extension $k / F$ is of conductor $f=4\left(\gamma_{2}-\gamma_{3}\right)^{2}=4\left(4-3 \gamma^{2}\right)$, where $4-3 \gamma^{2}$ generates the unique unramified prime above 23 in $F$. To determine the resolvent field $k^{\prime}$ from classfield theory using (11), let $\chi$ and $\psi$ be the quadratic numerical characters of $F$ realized modulo $4\left(4-3 \gamma^{2}\right)$ which are induced by extending the Kronecker symbols $\left(\frac{\gamma}{\mu}\right)$ and $\left(\frac{4-3 \gamma^{2}}{\mu}\right)$, and setting

$$
\chi(\eta)=\left(\frac{\gamma}{(\eta)}\right) \quad \text { and } \quad \psi(\eta)=\left(\frac{4-3 \gamma^{2}}{(\eta)}\right) \quad \text { for } \eta>0 .
$$

Since $\chi$ and $\psi$ annihilate $U_{F}^{+}$and $h^{+}=1$, it follows from classfield 
theory (chiefly Theorem $3[4])$ that

$$
\begin{aligned}
& {[1]=\{(\eta) \mid \chi \circ \psi(\eta)=1, \eta>0\} \quad \text { and }} \\
& {[\tau]=\{(\eta) \mid \chi \circ \psi(\eta) \neq 1, n>0\}}
\end{aligned}
$$

in (9) where $m=\left(4\left(4-3 \gamma^{2}\right)\right)$. Let $\rho$ be the quadratic numerical character of $F$, realized modulo 4 , satisfying $\rho(\eta) \equiv N_{F / Q} \eta(\bmod 4)$. Then we find the sets $[\phi]$ in (11) are

$$
\begin{aligned}
{\left[\phi_{1,1}\right] } & =\{(\eta) \mid \chi \circ \psi(\eta)=1, \quad \rho(\eta)=1 \text { and } \eta>0\} \quad \text { and } \\
{\left[\phi_{\tau,-1}\right] } & =\{(\eta) \mid \chi \circ \psi(\eta) \neq 1, \quad \rho(\eta) \neq 1 \text { and } \eta>0\} .
\end{aligned}
$$

In particular, we have $\mathscr{B}=\{(\eta) \mid \chi \circ \psi \circ \rho(\eta)=1, \eta>0\}$ with corresponding classfield $k^{\prime}=F\left(i \gamma_{1}^{1 / 2}\left(\gamma_{2}-\gamma_{3}\right)\right)$ since $\rho(\eta)=(-1 /(\eta))$ for $n>0$.

The resolvent field $k^{\prime}$ for $p(x)$ over $F$ can be expressed as a compositum of resolvent fields (one for each of the splitting fields $K_{i}(1 \leq i \leq t)$ in (3)) in a manner consistent with the decomposition $k=\prod k_{i}$. To see this, let $W_{i}=\left\{\phi_{t, s} \mid s \equiv \theta(\tau) \bmod n_{i}\right\}$ with fixed field $k_{i}^{\prime}(1 \leq i \leq t)$. Now each $k_{i}^{\prime} \subset k_{i}\left(\zeta^{n / n_{\imath}}\right)$ since $k_{i}\left(\zeta^{n / n_{\imath}}\right)$ corresponds through Galois theory to the subgroup $\left\{\phi_{\tau, 1} \mid \theta(\tau) \equiv 1\right.$ $\left.\left(\bmod n_{i}\right)\right\} \quad(1 \leq i \leq t)$. Suppose $\alpha^{(i)}$ is a generator for $R_{i} / F$ (and thus for $\left.K_{i} / k_{i}\right)$ of zero trace in (5), and form the resolvents

$$
\begin{aligned}
\omega_{\nu}^{(i)}=\alpha^{(i)}+\zeta^{-\nu n / n_{i}} \sigma\left(\alpha^{(i)}\right)+\cdots+\zeta^{-\nu\left(n_{i}-1\right) n / n_{i}} \sigma^{\left(n_{i}-1\right)}\left(\alpha^{(i)}\right) & \\
& (\nu \in Z) .
\end{aligned}
$$

Set $\beta_{\nu}^{(i)}=\left(\omega_{\nu}^{(i)}\right)^{n}$ for $\nu \in Z$, and

$$
\alpha_{\mu}^{(i)}=\frac{1}{n_{i}} \sum_{\nu=1}^{n_{\imath}-1} \zeta^{\mu \nu n / n_{\iota}} \omega_{\nu}^{(i)}=\sigma^{\mu}\left(\alpha^{(i)}\right) \quad\left(\mu \in Z_{n_{\imath}}\right),
$$

for $1 \leq i \leq t$. Using an argument analogous to that in the proof of Lemma 1 , one finds the $\beta_{\nu}^{(i)}$ are fixed by each $\phi_{\tau, s}$ in $W_{i}$ and thus lie in $\left.k_{i}^{\prime}(1 \leq i \leq t)\right)$. The minimal polynomial $p_{i}(x)$ over $F$ for $\alpha^{(i)}$ has splitting field $K_{i}$ and resolvent field $k_{i}^{\prime}$ over $F$. Since $W=\bigcap W_{i}$, the resolvent field $k^{\prime}$ is the compositum $\prod_{i=1}^{t} k_{i}^{\prime}$. In addition, $\alpha=\prod \alpha^{(i)}$ is a generator for $R / F$ of zero trace.

In the next section we take up the construction of the fields $R_{i}$, determining suitable generators $\alpha^{(i)}$ and computing their minimal polynomial $p_{i}(x)$ over $F$. 
3. Construction of $K=k \cdot R$ when $[R: F]$ is a prime power. Here we retain the notation and setup of the previous sections, but with $n=l^{u}$ a prime power. In this section we shall describe how to construct arbitrary extensions $K=k \cdot R$ with $K \cap Q(\zeta)=Q$ and Galois group $G$ satisfying (1). (The methods can be adapted to construct such extensions $K$ where $K \cap Q(\zeta) \neq Q$, but require more delicate treatment.) Under the assumption $K \cap Q(\zeta)=Q$, one recalls that $G(k(\zeta) / F)=\left\{\phi_{\tau, s} \mid \tau \in V, s \in Z_{n}^{*}\right\}$, so that the values $\sum_{\nu \in Z_{n}^{*}} \zeta^{\mu \nu} \beta_{\nu}$ $(0 \leq \mu<n)$ are readily seen to all lie in $k$. In addition, we have

$$
\sigma^{\lambda}\left(\omega_{\nu}\right)=\zeta^{\nu \lambda} \omega_{\nu} \quad(\nu \in Z)
$$

in (5). We may further assume that $\omega_{\nu}=0$ for $(\nu, n)>1$ by replacing $\alpha$ with $\alpha-\frac{1}{l}\left(\alpha_{0}+\alpha_{l^{u-1}}+\cdots+\alpha_{(l-1) l^{u-1}}\right)$ if necessary in (5), since

$$
\begin{aligned}
\omega_{\nu}\left(\alpha-\frac{1}{l} \sum_{t=0}^{l-1} \alpha_{t l^{u-1}}\right) & \left.=\omega_{\nu}\left(\alpha-\frac{1}{l} \sum_{t=0}^{l-1} \sigma^{t l^{u-1}}(\alpha)\right)\right) \\
& =\omega_{\nu}(\alpha)-\omega_{\nu}(\alpha)\left[\frac{1}{l} \sum_{t=0}^{l-1} \zeta^{\nu t l^{u-1}}\right]
\end{aligned}
$$

equals $\omega_{\nu}(\alpha)$ if $l \nmid \nu$ else zero when $l \mid \nu$ from (6) and (15). We shall henceforth assume that this normalization has been made. Finally we note that if $\tilde{\phi}$ is any extension of $\phi_{\tau, s}$ to $K(\zeta)$ which fixes $R$, then $\tilde{\phi}\left(\omega_{\nu}\right)=\omega_{\nu s \theta\left(\tau^{-1}\right)}$ from the proof of Lemma 1. Thus we find that $\tilde{\alpha}=\frac{1}{n} \sum_{\nu \in Z_{n}^{*}} \omega_{\nu}^{r}$ lies in $R$; in fact, $R=F(\tilde{\alpha})$ if $(r, n)=1$.

We now describe how to construct arbitrary extensions $K=k \cdot R$ as above with $K \cap Q(\zeta)=Q$ and Galois group $G$ satisfying (1). Explicit examples will be constructed later in $\S 4$.

First choose an irreducible polynomial $q(x)$ of degree $\phi(n)$ over $F$ with Galois group isomorphic to $H=Z_{n}^{*}$ and splitting field $k^{\prime}$. Consider an explicit isomorphism

$$
\psi: G\left(k^{\prime} / F\right) \cong H
$$

and label the element $\rho_{r}$ of $G\left(k^{\prime} / F\right)$ so that $\phi\left(\rho_{r}\right)=r$. Choose a zero $\varepsilon$ of $q(x)$, and label its conjugates

$$
\varepsilon_{r}=\rho_{r}(\varepsilon) \quad(r \in H)
$$


Assume the $\varepsilon_{r}$ satisfy the condition

$\prod_{t \in H} \varepsilon_{t}^{r_{t}}$ is an $n$th power in $k^{\prime}$ only if the $r_{t}$ are

all congruent modulo $n$,

$$
\text { or } \sum_{t \in H} t r_{t} \equiv 0(\bmod n) \text { when } n=l \text {. }
$$

Finally, fix an ordering for the elements of $H=Z_{n}^{*}$. Select a function $f: H \rightarrow Z$ which sends a given residue class to some specified integer representing that class, and for which the group matrix

$$
A=\left(a_{s, t}\right) \quad(s, t \in H),
$$

with $a_{s, t}=f\left(s t^{-1}\right)$, has an inverse, say $B$. (We note that it is easy to find such functions $f$.) Set

$$
\beta_{\nu}=\prod_{t \in H} \varepsilon_{t}^{a_{\nu, t}} \quad(\nu \in H) .
$$

For this choice, note that $\rho_{r}\left(\beta_{\nu}\right)=\beta_{\nu r}$ for $\nu$ and $r$ in $H$, since

$$
\rho_{r}\left(\beta_{\nu}\right)=\prod_{t \in H} \rho_{r}\left(\varepsilon_{t}\right)^{\alpha_{\nu, t}}=\prod_{t \in H} \varepsilon_{r t}^{a_{\nu, t}}=\prod_{t \in H} \varepsilon_{r t}^{a_{\nu r, t r}}=\prod_{t \in H} \varepsilon_{t}^{a_{\nu r, t}} .
$$

With the above choices we have

Proposition 3. Suppose the elements $\beta_{\nu}(\nu \in H)$ are selected as in (20), where the $\varepsilon_{t}$ satisfy condition (18). Then the extension $k^{\prime}\left(\zeta, \beta_{\nu}^{1 / n} \mid \nu \in H\right)$ is cyclic of degree $n$ over $k^{\prime}(\zeta)$.

Proof. Note that $\prod_{\nu \in H} \beta_{\nu}^{r_{\nu}}$ is an $n$th power in $k^{\prime}$

if and only if $\prod_{\nu \in H} \prod_{t \in H} \varepsilon_{t}^{a_{\nu, t}^{r_{\nu}}}$ is an $n$th power in $k^{\prime}$

if and only if $\prod_{t \in H} \varepsilon_{t}^{\sum_{\nu \in H} a_{\nu, t_{\nu}} r_{\nu}}$ is an $n$th power in $k^{\prime}$.

Since $\sum_{\nu \in H} a_{\nu, t} r_{\nu} \equiv \sum_{\nu \in H} \nu t^{*} r_{\nu} \equiv t^{*} \sum_{\nu \in H} \nu r_{\nu}(\bmod n)$ for $t \in H$ from (19), it follows from (18) and (20) that $\prod_{\nu \in H} \beta_{\nu}^{r_{\nu}}$ is an $n$th power in $k^{\prime}$ if and only if

$$
\sum_{\nu \in H} \nu r_{\nu} \equiv 0 \quad(\bmod n)
$$

It follows from (21) that for a given $\nu \in H$,

$$
\beta_{\nu}=\beta_{1}^{\nu} \cdot \gamma^{n}
$$

for some $\gamma$ in $k^{\prime}$. Also $\beta_{1}^{r_{1}}$ lies in $\left(k^{\prime}\right)^{n}$ if and only if $r_{1} \equiv 0$ $(\bmod n)$. In particular, $k^{\prime}\left(\zeta, \beta_{\nu}^{1 / n} \mid \nu \in H\right)=k^{\prime}\left(\zeta, \beta_{1}^{1 / n}\right)$, and the conclusion of the proposition follows. 
To proceed with the construction, fix a generator $\sigma$ for $k^{\prime}\left(\zeta, \beta_{\nu}^{1 / n} \mid \nu \in H\right)$ over $k^{\prime}(\zeta)$. It follows from the proposition above that $\beta_{t}^{1 / n}$ generates $k^{\prime}\left(\zeta, \beta_{\nu}^{1 / n} \mid \nu \in H\right)$ over $k^{\prime}(\zeta)$ for any $t$ in $H$. Choose particular $n$th roots $\beta_{t}^{1 / n}$ so that $\sigma\left(\beta_{t}^{1 / n}\right)=\zeta^{t} \beta_{t}^{1 / n}(t \in H)$, and set

$$
\alpha_{\mu}=\frac{1}{n} \sum_{\substack{t=1 \\(t, n)=1}} \zeta^{\mu t} \beta_{t}^{1 / n} \quad(\mu \in Z) .
$$

The values $\alpha_{\mu}$ in (23) are distinct and comprise a complete set of conjugates over $F$. In particular $\alpha=\alpha_{0}$ generates an extension $R$ over $F$ of degree $n$ with Lagrange resolvents (5) satisfying

$$
\omega_{\nu}= \begin{cases}\beta_{\nu}^{1 / n} & \text { if } l \nmid \nu, \\ 0 & \text { if } l \mid \nu .\end{cases}
$$

The minimal polynomial $p(x)$ for $\alpha$ has splitting field $K$, which we assert is the compositum $k \cdot R$ for a certain abelian extension $k / F$. We shall determine the abelian extension $k$ from classfield theory. Suppose $k^{\prime} / F$ has conductor $f^{\prime}$ and $m=L C M\left(f^{\prime}, n O_{F}\right)$. If $\mathscr{C}$ is the group of fractional $F$-ideals prime to $m$, then $k^{\prime} / F$ corresponds through classfield theory to a subgroup $\mathscr{B}$ of index $\phi(n)$ in $\mathscr{C}$. In particular, $\mathscr{C} / \mathscr{B} \cong G\left(k^{\prime} / F\right)$ via the Artin map

$$
[r] \rightarrow \rho_{r} \quad(r \in H),
$$

where $[r]$ denotes the coset of $\mathscr{C} / \mathscr{B}$ set to $\rho_{r}$ in $G\left(k^{\prime} / F\right)$. We assume that the isomorphism $\psi$ in (16) is chosen so that the sets

$$
\mathscr{B}_{r}=\{\alpha \in[r] \mid \mathcal{N} a \equiv r \bmod n\} \neq \varnothing
$$

for each $r$ in $H$. (Note that this can always be arranged; in the extreme case $k^{\prime}=F(\zeta)$, the map $\psi$ is the inverse of the Artin map of $F(\zeta) / F)$. The following result specifies how to obtain $k$ and determine $G=G(K / F)$.

THeOREM 1. The set $\mathscr{A}=\bigcup_{r \in H} \mathscr{B}_{r}$ is a subgroup of $\mathscr{C}$ with corresponding classfield $k / F$. The splitting field $K=k \cdot R$ with Galois group $G$, a semi-direct product of $T$ by $V$, where $T=\left\langle\left.\sigma\right|_{K}\right\rangle$ has fixed field $k$ and $V=G(K / R)$ is isomorphic to a subgroup of $Z_{n}^{*}$. Furthermore, the group $G$ satisfies (1), where for any $\tau$ in $V$, the value $\theta(\tau)$ is the unique $s$ in $H$ for which $\left[\phi_{\tau, s}\right] \subset B$ in (11)

Proof. Evidently $\mathscr{A}$ is a subgroup of $\mathscr{C}$, and thus corresponds through classfield theory to an abelian extension $k / F$. In addition, 
$\mathscr{A} \cap \mathscr{B}=\mathscr{B}_{1}=\left[\phi_{1,1}\right]$ so the corresponding classfields $k \cdot k^{\prime}=k^{\prime}(\zeta)=$ $k(\zeta)$. Since $\mathscr{A} \mathscr{B}=\mathscr{C}$ we have the following diagram of subfields of $k(\zeta)$ and corresponding subgroups of $\mathscr{C}$ :
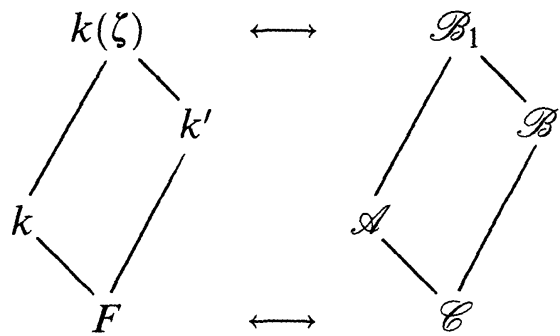

In particular, $[k(\zeta): k]=\left[\mathscr{A}: \mathscr{B}_{1}\right]=\phi(n)$ so $k \cap F(\zeta)=F$ and $G(k / F) \cong G\left(k^{\prime}(\zeta) / k^{\prime}\right) \cong \mathscr{B} / \mathscr{B}_{1}$. Since $\mathscr{B}_{r} \neq \varnothing$ and $[r] \rightarrow \rho_{r}$ via the Artin map of $k^{\prime} / F$, there exists an extension $\bar{\rho}_{r}$ of $\rho_{r}$ to $k^{\prime}(\zeta)=$ $k(\zeta)$, in fact to $K(\zeta)$, which maps

$$
\zeta \rightarrow \zeta^{r} \quad \text { and } \quad \omega_{\nu} \rightarrow \omega_{\nu r} \quad(r \in H) .
$$

Now $K$ is the field fixed by the $\bar{\rho}_{r}(r \in H)$. But since the $\bar{\rho}_{r}$ fix $k$, it follows that $k \subset K$, in fact, with $G(K / k)=\left\langle\left.\sigma\right|_{K}\right\rangle$. Also $K \cap F(\zeta)=$ $F$. In particular, we have the following correspondence from Galois theory
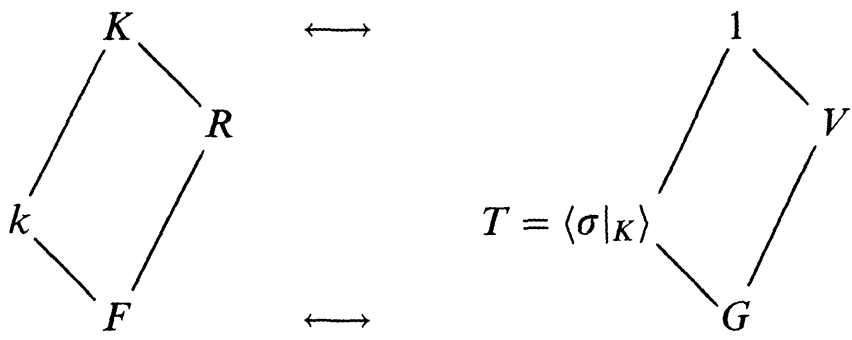

Since no $\left.\sigma\right|_{K} \neq 1$ fixes $R$, we find that $K=k \cdot R$ and $V=G(K / R) \cong$ $G(k / F)$. It follows that $G$ is a semi-direct product of $T$ by $V$, so that the relation

$$
\left.\tau \sigma\right|_{K} \tau^{-1}=\left.\sigma\right|_{K} ^{\theta(\tau)} \quad(\tau \in V)
$$

defines a homomorphism $\theta: V \rightarrow Z_{n}^{*}$ as in (1). The map $\theta$ is injective since $K$ is the splitting field for $R$; thus $V$ is isomorphic to some subgroup of $Z_{n}^{*}$.

To complete the proof of the theorem it remains to specify the range of $\theta$. Recall from (11) how $\mathscr{B}$ is constructed from certain cosets $[\phi]$ in $\mathscr{C}$. It follows from (10) that for a given $\tau$ in $V$, the set $[\tau] \cap \mathscr{B}$ coincides with exactly one of the cosets $\left[\phi_{\tau, s}\right](s \in H)$; namely, that for which $s=\theta(\tau)$. 
To aid in the computation of $k$, we introduce the following corollary.

COROllary 1. Let $h(x)$ be any irreducible polynomial of degree $\phi(n)$ over $F$ with splitting field $k^{\prime}$. Select a zero $\delta$ of $h(x)$ and label its conjugates

$$
\delta_{r}=\rho_{r}(\delta) \quad(r \in H)
$$

as in (17). Then the elements $N_{\nu}=\sum_{\mu \in H} \zeta^{\mu \nu} \delta_{\mu}(\nu \in H)$ all lie in the classfield $k$.

Proof. Since $N_{\nu} \in k(\zeta)$ it suffices to show that each map $\bar{\rho}_{r}$ $(r \in H)$, described by (27), fixes $N_{\nu}$. But $\bar{\rho}_{r}\left(N_{\nu}\right)=\sum_{\mu \in H} \zeta^{\mu \nu r} \delta_{\mu r}=$ $\sum_{\mu \in H} \zeta^{\mu \nu} \delta_{\mu}$ for any $r, \nu$ in $H$.

To compute the minimal polynomial

$$
p(x)=x^{n}+a_{2} x^{n-2}+\cdots+a_{n}
$$

for $\alpha=\alpha_{0}$ in (6), we introduce a counting function

$$
N\left(x_{1}, \ldots, x_{t}\right)=\sum^{\prime} \zeta^{x_{1} u_{1}+\cdots+x_{t} u_{t}}
$$

for non-negative integers $x_{1}, \ldots, x_{t}$. The primed sum is taken over all $t$-tuples $\left(u_{1}, \ldots, u_{t}\right)$ with integral components $u_{i}(1 \leq i \leq t)$ pairwise mutually distinct and in the range $[0, n)$. The counting function (30) has the following iterative property.

Proposition 4.

$$
\dot{N}\left(x_{1}\right)= \begin{cases}n & \text { if } x_{1} \equiv 0(\bmod n), \\ 0 & \text { if } x_{1} \neq \equiv(\bmod n) .\end{cases}
$$

For $t>1$,

$$
\text { (32) } N\left(x_{1}, \ldots, x_{t}\right)=\left\{\begin{array}{l}
(n-t+1) N\left(x_{1}, \ldots, x_{t-1}\right) \\
\text { if } x_{t} \equiv 0(\bmod n), \\
-N\left(x_{1}+x_{t}, \ldots, x_{t-1}\right) \\
-\cdots-N\left(x_{1}, \ldots, x_{t-1}+x_{t}\right) \quad \text { otherwise. }
\end{array}\right.
$$

Proof. Formula $(31)$ is obvious. To verify $(32)$ when $x_{t} \equiv 0(\bmod n)$, we first note that there are $n-t+1$ choices for the value $u_{t}$ in any tuple $\left(u_{1}, u_{2}, \ldots, u_{t}\right)$ in the primed sum, once the first $t-1$ components have been selected. Since $\zeta^{x_{1} u_{1}+\cdots+x_{t-1} u_{t-1}}=\zeta^{x_{1}} u_{1}+\cdots+x_{t} u_{t}$ in this 
case, evidently $N\left(x_{1}, \ldots, x_{t}\right)=(n-t+1) N\left(x_{1}, \ldots, x_{t-1}\right)$. Now if $x_{t} \neq 0(\bmod n)$ then $\sum_{u=0}^{n-1} \zeta^{u x_{t}}=0$. Since $\sum^{\prime} \zeta^{u_{1} x_{1}+\cdots+u_{t} x_{t}}$ equals

$$
\begin{gathered}
\sum^{\prime} \zeta^{u_{1} x_{1}+\cdots+u_{t-1} x_{t-1}}\left(\sum \zeta_{t}^{u_{t} x_{t}}\right)-\sum^{\prime} \zeta^{u_{1}\left(x_{1}+x_{t}\right)+\cdots+u_{t-1} x_{t-1}} \\
-\cdots-\sum^{\prime} \zeta^{u_{1} x_{1}+\cdots+u_{t-1}}\left(x_{t-1}+x_{t}\right)
\end{gathered}
$$

we find that $(32)$ holds when $x_{t} \not \equiv 0(\bmod n)$.

Corollary 2. (i) If $x_{1}+\cdots+x_{t} \not \equiv 0(\bmod n)$ then $N\left(x_{1}, \ldots, x_{t}\right)$ $=0$.

(ii) If $x_{1}+\cdots+x_{t}$ sums to zero $(\bmod n)$ but no smaller subset sums to zero $(\bmod n)$ then $N\left(x_{1}, \ldots, x_{t}\right)=(-1)^{t-1}(t-1) ! n$ for $t \geq 1$.

We shall omit the proof of the corollary since both (i) and (ii) follow easily from (31) and (32) by using induction on $t$.

We are ready to determine the coefficient of $p(x)$.

THEOREM 2. The coefficient $a_{m}(2 \leq m \leq n)$ for the term $x^{n-m}$ is a sum of all terms of the form

$$
\frac{(-1)^{m}}{n^{m}} \beta_{t_{1}}^{c_{1} / n} \cdots \beta_{t_{e}}^{c_{e} / n} N(\underbrace{t_{1}, \ldots, t_{1}}_{c_{1} \text { times }}, \ldots, \underbrace{t_{e}, \ldots, t_{e}}_{c_{e} \text { times }}) /\left(c_{1} ! \cdots c_{e} !\right)
$$

where $t_{i} \in H, 1 \leq c_{i} \leq m$ for $1 \leq i \leq e$ with $\sum_{i=1}^{e} c_{i}=m$ and $\sum_{i=1}^{e} c_{i} t_{i} \equiv 0(\bmod n)$. Each term $\beta_{t_{1}}^{c_{1} / n} \ldots \beta_{t_{e}}^{c_{e} / n}$ which appears is a product of the elements $\varepsilon_{r}$.

Proof. Now

$$
p(x)=\prod_{\mu=0}^{n-1}\left(x-\alpha_{\mu}\right)=\prod_{\mu=0}^{n-1}\left(x-\frac{1}{n} \sum \zeta^{\mu t} \beta_{t}^{1 / n}\right),
$$

where the sum is over $t$ in $H$. Expanding the product, we find that each term that appears in the expressions for $a_{m}$ is of the form $\beta_{t_{1}}^{c_{1} / n} \cdots \beta_{t_{e}}^{c_{e} / n}$, where $c_{1}+\cdots+c_{e}=m$, with multiplier

$$
\frac{(-1)^{m}}{n^{m}} \sum^{\prime} \zeta^{\left(u_{1,1}+\cdots+u_{1, c_{1}}\right) t_{1}+\cdots+\left(u_{e, 1}+\cdots+u_{e, c_{e}}\right) t_{e}} \text {. }
$$

Here the primed sum is over $m$-tuples $\left(u_{1,1}, \ldots, u_{e, c_{e}}\right)$ in $[0, n)^{m}$ with mutually distinct components and ordered so

$$
u_{1,1}<u_{1,2}<\cdots<u_{1, c_{1}}, \ldots, u_{e, 1}<u_{e, 2}<\cdots<u_{e, c_{e}} .
$$


But (34) equals the multiplier of $\beta_{t_{1}}^{c_{1} / n} \ldots \beta_{t_{e}}^{c_{e} / n}$ in (33) above, and the terms for which $\sum_{i=1}^{e} c_{i} t_{i} \not \equiv 0(\bmod n)$ have multiplier equal zero by Corollary 2 . The terms with $\sum_{i=1}^{e} c_{i} t_{i} \equiv 0(\bmod n)$ satisfy

$$
\beta_{t_{1}}^{c_{1} / n} \cdots \beta_{t_{e}}^{c_{e} / n}=\prod_{i=1}^{e} \prod_{\nu \in H} \varepsilon_{\nu}^{a_{t_{l}, \nu} c_{i} / n}=\prod_{\nu \in H} \varepsilon_{\nu}^{r_{\nu}}
$$

where

$$
r_{\nu}=\frac{1}{n} \sum_{i=1}^{e} a_{t_{i}, \nu} c_{i}
$$

But $a_{t_{l}, \nu} \equiv t_{i} \nu^{*}(\bmod n)$ so $\sum_{i=1}^{e} a_{t_{t}, \nu} c_{i} \equiv \nu^{*} \sum_{i=1}^{e} t_{i} c_{i} \equiv 0(\bmod n)$; hence the $r_{\nu}$ are integers in (35). (Here $\nu^{*}$ is the multiplicative inverse of $\nu(\bmod n))$.

For $n=3,4,5,7$ and 8 and indicated choice of matrix $A$ in (19), we obtain the following expressions for the coefficients of $p(x)$. (Here $N$ and $\operatorname{Tr}$ denote the norm and trace from $k^{\prime}$ to $F$ respectively.)

(37) $n=3$ :

$$
\begin{gathered}
A=\left[\begin{array}{ll}
1 & 2 \\
2 & 1
\end{array}\right], \quad B=\frac{1}{3}\left[\begin{array}{cc}
-1 & 2 \\
2 & -1
\end{array}\right], \\
a_{2}=-(N \varepsilon) / 3, \quad a_{3}=-(N \varepsilon)(\operatorname{Tr} \varepsilon) / 27
\end{gathered}
$$

(38) $n=4$ :

$$
\begin{gathered}
A=\left[\begin{array}{ll}
1 & 3 \\
3 & 1
\end{array}\right], \quad B=\frac{1}{8}\left[\begin{array}{cc}
-1 & 3 \\
3 & -1
\end{array}\right], \\
a_{2}=-(N \varepsilon) / 4, \quad a_{3}=0, \quad a_{4}=\left(2 N \varepsilon^{2}-\operatorname{Tr} \beta\right) / 64
\end{gathered}
$$

(39) $n=5$ :

$$
\begin{aligned}
A= & {\left[\begin{array}{cccc}
1 & 3 & -1 & 2 \\
2 & 1 & 3 & -1 \\
-1 & 2 & 1 & 3 \\
3 & -1 & 2 & 1
\end{array}\right], \quad B=\frac{1}{5}\left[\begin{array}{cccc}
1 & 0 & -1 & 1 \\
1 & 1 & 0 & -1 \\
-1 & 1 & 1 & 0 \\
0 & -1 & 1 & 1
\end{array}\right] } \\
& a_{2}=-\left(\varepsilon_{1} \varepsilon_{4}+\varepsilon_{2} \varepsilon_{3}\right) / 5, \quad a_{3}=-\operatorname{Tr}\left(\varepsilon_{1} \varepsilon_{2} \varepsilon_{3}\right) / 25 \\
& a_{4}=-\left(\operatorname{Tr}\left(\varepsilon_{1} \varepsilon_{2}^{2} \varepsilon_{3}\right)+N \varepsilon-\left(\varepsilon_{1}^{2} \varepsilon_{4}^{2}+\varepsilon_{2}^{2} \varepsilon_{3}^{2}\right)\right) / 125 \\
& a_{5}=-\left(\operatorname{Tr}\left(\beta-5 \varepsilon_{1} \varepsilon_{2}^{2} \varepsilon_{3}^{2}\right)+5(N \varepsilon) \cdot \operatorname{Tr} \varepsilon\right) / 3125
\end{aligned}
$$


(40) $n=7$ :

$$
\begin{aligned}
& A=\left[\begin{array}{cccccc}
1 & -2 & 4 & -1 & 2 & 3 \\
3 & 1 & -2 & 4 & -1 & 2 \\
2 & 3 & 1 & -2 & 4 & -1 \\
u-1 & 2 & 3 & 1 & -2 & 4 \\
4 & -1 & 2 & 3 & 1 & -2 \\
-2 & 4 & -1 & 2 & 3 & 1
\end{array}\right], \\
& B=\frac{1}{7}\left[\begin{array}{cccccc}
0 & 1 & 1 & 0 & 0 & -1 \\
-1 & 0 & 1 & 1 & 0 & 0 \\
0 & -1 & 0 & 1 & 1 & 0 \\
0 & 0 & -1 & 0 & 1 & 1 \\
1 & 0 & 0 & -1 & 0 & 1 \\
1 & 1 & 0 & 0 & -1 & 0
\end{array}\right],
\end{aligned}
$$

$$
\begin{aligned}
& a_{2}=-\left(\varepsilon_{1} \varepsilon_{6}+\varepsilon_{2} \varepsilon_{5}+\varepsilon_{3} \varepsilon_{4}\right) / 7 \text {, } \\
& a_{3}=-\left[2\left(\varepsilon_{1} \varepsilon_{2} \varepsilon_{4}+\varepsilon_{3} \varepsilon_{5} \varepsilon_{6}\right)+\operatorname{Tr}\left(\varepsilon_{2} \varepsilon_{4} \varepsilon_{5}\right)\right] / 49 \text {, } \\
& a_{4}=-\left[-\left(\varepsilon_{2} \varepsilon_{3} \varepsilon_{4} \varepsilon_{5}+\varepsilon_{1} \varepsilon_{2} \varepsilon_{5} \varepsilon_{6}+\varepsilon_{1} \varepsilon_{3} \varepsilon_{4} \varepsilon_{6}\right)\right. \\
& \left.+\operatorname{Tr}\left(\varepsilon_{1} \varepsilon_{4} \varepsilon_{5} \varepsilon_{2}^{2} / \varepsilon_{3}+3 \varepsilon_{1} \varepsilon_{2} \varepsilon_{4} \varepsilon_{5}\right)-2\left(\varepsilon_{2}^{2} \varepsilon_{5}^{2}+\varepsilon_{1}^{2} \varepsilon_{6}^{2}+\varepsilon_{3}^{2} \varepsilon_{4}^{2}\right)\right] / 343, \\
& a_{5}=-\left[\operatorname { T r } \left(\varepsilon_{1} \varepsilon_{4} \varepsilon_{5}^{2} \varepsilon_{2}^{2} / \varepsilon_{3}+2 \varepsilon_{1} \varepsilon_{2}^{2} \varepsilon_{4}^{2} \varepsilon_{5} / \varepsilon_{6}\right.\right. \\
& \left.\left.-3 \varepsilon_{2}^{2} \varepsilon_{4} \varepsilon_{5}^{2}-\varepsilon_{1} \varepsilon_{5}^{2} \varepsilon_{2} \varepsilon_{6}-2 \varepsilon_{1} \varepsilon_{2}^{2} \varepsilon_{4} \varepsilon_{5}\right)+5(N \varepsilon) \operatorname{Tr}\left(\varepsilon^{-1}\right)\right] / 2401, \\
& a_{6}=-7^{-5}\left[\operatorname { T r } \left(\varepsilon_{1} \varepsilon_{4}^{2} \varepsilon_{5}^{2} \varepsilon_{2}^{3} / \varepsilon_{3} \varepsilon_{6}-\varepsilon_{2}^{2} \varepsilon_{5}^{2} \varepsilon_{4}^{2}-2 \varepsilon_{1} \varepsilon_{4} \varepsilon_{5}^{2} \varepsilon_{2}^{3} / \varepsilon_{3}+3 \varepsilon_{1} \varepsilon_{2} \varepsilon_{5}^{2} \varepsilon_{4} \varepsilon_{6}\right.\right. \\
& +3 \varepsilon_{1}^{2} \varepsilon_{2}^{2} \varepsilon_{4} \varepsilon_{5} \varepsilon_{6} / \varepsilon_{3}-\varepsilon_{1} \varepsilon_{2}^{2} \varepsilon_{4} \varepsilon_{5}^{2}-\varepsilon_{1} \varepsilon_{2}^{2} \varepsilon_{5} \varepsilon_{4}^{2} \\
& \left.+2 \varepsilon_{1} \varepsilon_{2} \varepsilon_{3} \varepsilon_{4}^{2} \varepsilon_{5}-5 \varepsilon_{1}^{2} \varepsilon_{2} \varepsilon_{4} \varepsilon_{5} \varepsilon_{6}\right) \\
& +\left(\varepsilon_{2}^{3} \varepsilon_{5}^{3}+\varepsilon_{1}^{3} \varepsilon_{6}^{3}+\varepsilon_{3}^{3} \varepsilon_{4}^{3}\right)+\left(\varepsilon_{1}^{2} \varepsilon_{2}^{2} \varepsilon_{4}^{2}+\varepsilon_{3}^{2} \varepsilon_{5}^{2} \varepsilon_{6}^{2}\right) \\
& +2\left(\varepsilon_{1} \varepsilon_{2}^{2} \varepsilon_{5}^{2} \varepsilon_{6}+\varepsilon_{2} \varepsilon_{3}^{2} \varepsilon_{4}^{2} \varepsilon_{5}+\varepsilon_{3} \varepsilon_{1}^{2} \varepsilon_{6}^{2} \varepsilon_{4}\right) \\
& \left.-5\left(\varepsilon_{2}^{2} \varepsilon_{3} \varepsilon_{4} \varepsilon_{5}^{2}+\varepsilon_{1}^{2} \varepsilon_{2} \varepsilon_{5} \varepsilon_{6}^{2}+\varepsilon_{3}^{2} \varepsilon_{1} \varepsilon_{6} \varepsilon_{4}^{2}\right)+15(N \varepsilon)\right],
\end{aligned}
$$$$
a_{7}=7^{-7}\left[\operatorname { T r } \left(-\beta+7 \varepsilon_{1} \varepsilon_{5}^{3} \varepsilon_{4} \varepsilon_{2}^{3} / \varepsilon_{3}+7 \varepsilon_{1} \varepsilon_{4}^{2} \varepsilon_{5}^{2} \varepsilon_{2}^{3} / \varepsilon_{3}-14 \varepsilon_{1} \varepsilon_{2}^{2} \varepsilon_{5}^{2} \varepsilon_{4}^{2}\right.\right.
$$$$
-7 \varepsilon_{1} \varepsilon_{2}^{3} \varepsilon_{4}^{2} \varepsilon_{5}^{2} / \varepsilon_{6}-7 \varepsilon_{1}^{2} \varepsilon_{2}^{2} \varepsilon_{4} \varepsilon_{5}^{2} \varepsilon_{6} / \varepsilon_{3}-7 \varepsilon_{1}^{2} \varepsilon_{2}^{3} \varepsilon_{4}^{2} \varepsilon_{5} / \varepsilon_{3}
$$$$
-7 \varepsilon_{2}^{3} \varepsilon_{4} \varepsilon_{5}^{3}-7 \varepsilon_{1} \varepsilon_{2}^{2} \varepsilon_{4} \varepsilon_{5}^{2} \varepsilon_{6}+21 \varepsilon_{1}^{2} \varepsilon_{2}^{2} \varepsilon_{4}^{2} \varepsilon_{5}+21 \varepsilon_{2}^{2} \varepsilon_{5}^{2} \varepsilon_{4}^{2} \varepsilon_{3}
$$$$
+21 \varepsilon_{1} \varepsilon_{2}^{3} \varepsilon_{4} \varepsilon_{5}^{2}+7 \varepsilon_{1} \varepsilon_{2}^{2} \varepsilon_{3} \varepsilon_{4}^{3} \varepsilon_{5} / \varepsilon_{6}+7 \varepsilon_{1}^{2} \varepsilon_{2} \varepsilon_{4} \varepsilon_{5}^{2} \varepsilon_{6}
$$$$
-14 \varepsilon_{1} \varepsilon_{2}^{2} \varepsilon_{5}^{3} \varepsilon_{6}-14 \varepsilon_{1} \varepsilon_{2}^{2} \varepsilon_{4}^{2} \varepsilon_{5} \varepsilon_{6}
$$

$$
\begin{aligned}
-35 \varepsilon_{1} \varepsilon_{2}^{2} \varepsilon_{3} \varepsilon_{4}^{2} \varepsilon_{5} & \left.+7 \varepsilon_{1} \varepsilon_{2}^{2} \varepsilon_{3} \varepsilon_{4} \varepsilon_{5}^{2}\right) \\
& +14(N \varepsilon) \operatorname{Tr} \varepsilon]
\end{aligned}
$$


(41) $n=8$ :

$$
\begin{aligned}
& A=\left[\begin{array}{cccc}
9 & -3 & -1 & 3 \\
-3 & 9 & 3 & -1 \\
-1 & 3 & 9 & -3 \\
3 & -1 & -3 & 9
\end{array}\right], \quad B=\frac{1}{64}\left[\begin{array}{cccc}
9 & 3 & -1 & -3 \\
3 & 9 & -3 & -1 \\
-1 & -3 & 9 & 3 \\
-3 & -1 & 3 & 9
\end{array}\right] \\
& a_{2}=-\left(\varepsilon_{1} \varepsilon_{7}+\varepsilon_{3} \varepsilon_{5}\right) / 8, \quad a_{3}=a_{5}=a_{7}=0, \\
& a_{4}=\left(-2 \operatorname{Tr}\left(\varepsilon_{1}^{3} \varepsilon_{3}\right)+5\left(\varepsilon_{1}^{2} \varepsilon_{7}^{2}+\varepsilon_{3}^{2} \varepsilon_{5}^{2}\right)\right. \\
& \left.-3\left(\varepsilon_{1}^{3} \varepsilon_{3}^{3} / \varepsilon_{5} \varepsilon_{7}+\varepsilon_{5}^{3} \varepsilon_{7}^{3} / \varepsilon_{1} \varepsilon_{3}\right)+4 N \varepsilon\right) / 1024 \\
& a_{6}=8^{-5}\left[\operatorname{Tr}\left(-\varepsilon_{1}^{6} \varepsilon_{3}^{3} / \varepsilon_{5}^{2} \varepsilon_{7}+3 \varepsilon_{1} \varepsilon_{5} \varepsilon_{7}^{4}-2 \varepsilon_{1}^{2} \varepsilon_{7} \varepsilon_{3}^{3}+2 \varepsilon_{1}^{4} \varepsilon_{3}^{3} / \varepsilon_{5}\right)\right. \\
& \left.-2(N \varepsilon)\left(\varepsilon_{1} \varepsilon_{7}+\varepsilon_{3} \varepsilon_{5}\right)-2\left(\varepsilon_{1}^{3} \varepsilon_{7}^{3}+\varepsilon_{3}^{3} \varepsilon_{5}^{3}\right)\right], \\
& a_{8}=8^{-8}\left[\operatorname { T r } \left(-\beta-20 \varepsilon_{1}^{5} \varepsilon_{7} \varepsilon_{3}^{3} / \varepsilon_{5}-8 \varepsilon_{1}^{5} \varepsilon_{7}^{2} \varepsilon_{3}-8 \varepsilon_{1}^{6} \varepsilon_{3}^{4} / \varepsilon_{5} \varepsilon_{7}+16 \varepsilon_{1}^{3} \varepsilon_{7}^{2} \varepsilon_{3}^{3}\right.\right. \\
& \left.+8 \varepsilon_{1}^{7} \varepsilon_{3}^{3} / \varepsilon_{5}^{2}+4 \varepsilon_{1}^{6} \varepsilon_{3}^{2}\right) \\
& +2\left(\varepsilon_{1}^{4} \varepsilon_{7}^{4}+\varepsilon_{3}^{4} \varepsilon_{5}^{4}\right)+2\left(\varepsilon_{1}^{6} \varepsilon_{3}^{6} / \varepsilon_{5}^{2} \varepsilon_{7}^{2}+\varepsilon_{5}^{6} \varepsilon_{7}^{6} / \varepsilon_{1}^{2} \varepsilon_{3}^{2}\right) \\
& -56\left(N \varepsilon^{2}\right)+32(N \varepsilon)\left(\varepsilon_{1}^{2} \varepsilon_{7}^{2}+\varepsilon_{3}^{2} \varepsilon_{5}^{2}\right) \\
& +32(N \varepsilon)\left(\varepsilon_{1}^{3} \varepsilon_{3}^{3} / \varepsilon_{5} \varepsilon_{7}+\varepsilon_{5}^{3} \varepsilon_{7}^{3} / \varepsilon_{1} \varepsilon_{3}\right)-8(N \varepsilon) \operatorname{Tr} \varepsilon_{1}^{3} \varepsilon_{3} \\
& \left.-6(N \varepsilon)\left(\varepsilon_{3}^{2} \varepsilon_{7}^{2}+\varepsilon_{1}^{2} \varepsilon_{5}^{2}\right)\right] \text {. }
\end{aligned}
$$

It is natural to ask how general is the above construction. In particular, suppose we are given an extension $K=k \cdot R$ of the kind under consideration and a generator $\alpha$ for $R$ over $F$ normalized so that the Lagrange resolvents (5) satisfy $\omega_{\nu}=0$ for $(\nu, n)>1$. Does the construction actually yield the generator $\alpha=\frac{1}{n} \sum_{\nu=1 ;(\nu, n)=1}^{n} \omega_{\nu}$ for a suitable choice of elements $\varepsilon_{r}$ in (17)? If not, to what extent can this be done?

We assert that a generator $\tilde{\alpha}=\frac{1}{n} \sum_{\nu=1 ;(\nu, n)=1}^{n}\left(\omega_{\nu}(\alpha)\right)^{r}$ for $R$ over $F$ is obtainable provided the inverse $B$ for the matrix $A$ in (19) has

$$
\operatorname{den} B=r \cdot n \quad \text { with }(r, n)=1 .
$$

(Here den $B$ denotes the least common denominator of the entries of $B$ when written in reduced form.)

Indeed, suppose $f$ is chosen so that the inverse matrix $B=\left(b_{s, t}\right)$ has $\operatorname{den} B=r n$ with $(r, n)=1$. Set

$$
\tilde{\varepsilon}_{\mu}=\prod_{t \in H} \omega_{t}^{r n b_{\mu, t}} \quad(\mu \in H) .
$$

It follows from the proof of Lemma 1 and (15), (19) and (42) that the $\tilde{\varepsilon}_{\mu}$ are fixed by $\sigma$ and $W$, so lie in $k^{\prime}$. Now, using (43) to obtain 
values $\tilde{\beta}_{\nu}$ from $(20)$; that is

$$
\tilde{\beta}_{\nu}=\prod_{\mu \in H} \tilde{\varepsilon}_{\mu}^{a_{\nu, \mu}} \quad(\nu \in H) .
$$

we find that

$$
\tilde{\beta}_{\nu}=\prod_{t \in H} \prod_{\mu \in H} \omega_{t}^{r n a_{\nu, \mu} b_{\mu, t}}=\prod_{t \in H} \omega_{t}^{r n\left(\sum_{\mu \in H} a_{\nu, \mu} b_{\mu, t}\right)}=\omega_{\nu}^{r n}=\beta_{\nu}^{r}
$$

for any $\nu$ in $H$. Thus the construction yields a generator $\tilde{\alpha}=\frac{1}{n} \sum \omega_{\nu}^{r}$ as claimed. In particular, the original generator $\alpha$ is obtained if the inverse matrix $B$ had den $B=n$. For these cases, the construction yields all generators $\alpha$ for $R$ normalized so $\omega_{\nu}=0$ for $(\nu, n) \neq 1$ in $(5)$.

The author has shown that condition (42) can be achieved if and only if $n=l^{u}$ is odd; moreover, that $\operatorname{den} B=n$ can be obtained at least for $n=3,5,7,9$ and 11 , but not for $n=13$. The proofs of these results involve long, tedious computations with group determinants and will appear elsewhere. (We note that the matrices $A$ in (37), (39) and (40) are so chosen that $\operatorname{den} B=n$ holds.)

Before concluding this section, some remarks on constructing extensions with prescribed ramification, e.g., classfields, are in order. For this purpose we require some results from local classfield theory. Let $L$ be any finite abelian extension of a number field $M$. Fix a prime ideal $\rho$ of $M$, and choose a prime $\mathscr{B}$ lying above $\rho$ in $L$. Let $L_{\mathscr{B}}$ and $M_{\mu}$ be the local extensions at $\mathscr{B}$ and $\rho$ respectively, and denote the corresponding unit groups by $U_{\mathscr{B}}$ and $U_{\mu}$. It is known [1, $10,11]$ from local classfield theory that the Galois group $G\left(L_{\mathscr{B}} / M_{p}\right)$ is isomorphic to $M_{\mu}^{x} / N_{L_{\mathscr{B}} / M_{\mu}}\left(L_{\mathscr{B}}^{x}\right)$, where $N_{L_{\mathscr{B}} / M_{\mu}}$ is the local norm map from $L_{\mathscr{B}}$ to $M_{\mu}$. In addition, the inertia group $I\left(L_{\mathscr{B}} / M_{\mu}\right)$ is isomorphic to $U_{\mu} / N_{L_{\mathscr{B}} / M_{\mu}}\left(U_{\mathscr{B}}\right)$, say of order $e(\mathscr{B}: \mu)$.

The construction detailed at the beginning of this section yields a generator $\alpha$ for a cyclic extension $K$ over $k$ of degree $n$ which is normal over $F$ and linearly disjoint from $Q(\zeta)$. Any $n$th root $\beta_{\nu}^{1 / n}$ generates $K(\zeta)$ over $k(\zeta)$. The idea here is to relate the ramification in $K / k$ to that in the Kummer extension $K(\zeta) / k(\zeta)$ where it is easy to determine. For convenience we state the relevant result of Kummer [10].

Proposition 5 (Kummer's criteria). Let $L=M\left(\beta^{1 / n}\right)$ for some number field $M$ which contains $\zeta$. Suppose $\rho$ is a prime ideal of $M$, 
say with uniformizing parameter $\pi$ in $M_{\mu}$. Write $\beta=\gamma \cdot \pi^{v}$ with $(\gamma, \pi)=1$ in $M_{\mu}$.

(i) If $(\rho, \beta)=(\rho, n)=1$, then $\rho$ is unramified in $L$.

(ii) If $(\rho, \beta)>1$ but $(\rho, n)=1$, then $\rho$ ramifies in $L$ if and only if $n \mid v$.

(iii) If $(\mu, n)>1$ but $(\mu, \beta)=1$, then $\rho$ ramifies in $L$ if and only if $\beta \notin U_{\mu}^{n}$.

(Note that determining whether $\beta$ lies in $U_{\mu}^{n}$ in statement (iii) amounts to checking whether $\beta$ is an nth power modulo a suitable power of $\mu$.)

Now fix a rational prime $p$ and choose a prime ideal $\widetilde{\mathscr{B}}$ lying above $p$ in $K(\zeta)$. Consider the following Hasse diagram of local extensions.

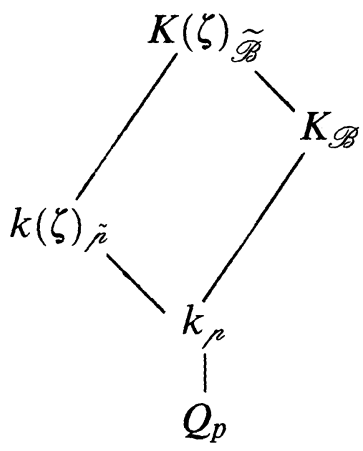

Here $\mathscr{B}=\widetilde{\mathscr{B}} \cap K, \tilde{\mu}=\widetilde{\mathscr{B}} \cap k(\zeta)$ and $\mu=\widetilde{\mathscr{B}} \cap k$. Consider the homomorphism $\Psi$ from unit quotient groups $U_{\tilde{\mu}} / N_{K(\zeta)_{\tilde{\mathscr{B}}}} / k(\zeta)_{\tilde{r}} U_{\widetilde{\mathscr{B}}}$ to $U_{\mu} / N_{K_{\mathscr{B}} / k_{\mu}} U_{\mathscr{B}}$ given by

$$
x \rightarrow N_{k(\zeta)_{\dot{\mu}} / k_{\mu}} x \cdot N_{K_{\mathscr{B}} / k_{\mu}} U_{\mathscr{B}} .
$$

The map $\Psi$ is injective. Indeed, $N_{k(\zeta)_{i} / k_{\mu}} x \in N_{K_{\mathscr{B}} / k_{\mu}} U_{\mathscr{B}}$ if and only if $x \in N_{k(\zeta)_{\tilde{\mu}} / k_{\mu}}^{-1}\left(N_{K_{\mathscr{B}} / k_{\mu}} U_{\mathscr{B}}\right)$. But, since $K \cap k(\zeta)=\hat{k}$, this last group $N_{k(\zeta)_{\tilde{i}} / k_{\mu}}^{-1}\left(N_{K_{\mathscr{B}} / k_{\mu}} U_{\mathscr{B}}\right)$ is readily seen, using the translation theorem of local classfield theory [11], to be norm group $N_{K(\zeta)_{\tilde{\mathscr{B}}} / k(\zeta)_{\tilde{i}}} U_{\widetilde{\mathscr{B}}}$. In particular, the ramification index $e(\widetilde{\mathscr{B}}: \tilde{\rho})$ divides $e(\mathscr{B}: \mu)$. In order for $\Psi$ to be onto, it is necessary that

$$
N_{k(\zeta)_{\tilde{r}} / k_{\mu}} U_{\tilde{\mu}} \cdot N_{K_{\mathscr{B}} / k_{\mu}} U_{\mathscr{B}}=U_{\mu} .
$$

In any case,

$$
e(\mathscr{B}: \mu)=e(\widetilde{\mathscr{B}}: \tilde{\mu}) \cdot\left[U_{\mu}: N_{k(\zeta)_{\tilde{r}} / k_{\mu}} U_{\tilde{\mu}} \cdot N_{K_{\mathscr{B}} / k_{\mu}} U_{\mathscr{B}}\right]
$$

For the situation at hand we have 
PROPOSITION 6. If $p \nmid l$ or $p=n=l$, then $U_{\tilde{r}} /\left(N_{K(\zeta) \tilde{\mathscr{A}}} / k(\zeta)_{\tilde{i}} U_{\widetilde{\mathscr{B}}}\right) \cong$ $U_{\mu} / N_{K_{\mathscr{G}} / k_{\mu}} U_{\mathscr{B}}$.

Proof. If $p \nmid l$ then $N_{k(\zeta)_{\dot{\mu}} / k_{\mu}} U_{\tilde{\mu}}=U_{\mu}$ since $k(\zeta) / k$ is unramified at $\mu$. If $p=n=l$ then $([K: k],[k(\zeta): k])=1$ so $e(\widetilde{\mathscr{B}}: \mu)=$ $e(\mathscr{B}: \mu) e(\tilde{\mu}: \mu)$. But $e(\widetilde{\mathscr{B}}: \rho)=e(\widetilde{\mathscr{B}}: \tilde{\mu}) e(\tilde{\mu}: \mu)$; hence $e(\widetilde{\mathscr{B}}: \tilde{\mu})=e(\mathscr{B}: \mu)$ so $(46)$ holds. In either case, $\Psi$ is an isomorphism.

It is clear from the above proposition that the problem to construct $K / k$ with specific ramification at $\mu$ is more one of finding $\beta_{\nu}$ in (20) (actually the $\varepsilon_{r}$ in (17)) so that $k\left(\zeta, \beta_{\nu}^{1 / n}\right) / k(\zeta)$ has the same ramification at $\tilde{\rho}$ by virtue of Kummer's criteria. Specifying the ramification at $\rho$ in the exceptional case $p=l$ and $n=l^{u}$ for $u>1$, requires more careful selection of $\beta$. We shall apply these ideas to construct some specific classfields among the examples given in the next section.

4. Some examples. Several examples are given now to exhibit the construction detailed in the previous section.

EXAMPLE 3. Next we wish to apply the construction to find a generator $\alpha$ and minimal polynomial for the unique cyclic field $K$, which is totally ramified at 7, unramified elsewhere, and of degree 7 over $Q$. Here $k^{\prime}=Q(\zeta)$ from Proposition 2 since $k=Q$. Selecting $\varepsilon=\varepsilon_{1}=\zeta^{-1}$ in (17) yields $\beta_{\nu}=\zeta^{\nu}$ in (20). The element $\beta=\beta_{1}=\zeta$ is a unit of $Q(\zeta)$, but not a seventh power in the completion of $Q(\zeta)$ at $(1-\zeta)$. Thus the extension $Q\left(\zeta, \beta^{1 / 7}\right) / Q(\zeta)$ is totally ramified at $(1-\zeta)$ and unramified elsewhere by Kummer's criteria. Since $e(\widetilde{\mathscr{B}}: \tilde{c})=e(\mathscr{B}: \mu)=7$ here, it follows that $K$ is generated by $7 \alpha=\zeta_{49}+\zeta_{49}^{-19}+\zeta_{49}^{-18}+\zeta_{49}^{18}+\zeta_{49}^{19}+\zeta_{49}^{-1}, \quad$ where $\zeta=\exp (2 \pi i / 49)$, with minimal polynomial $p(x)=x^{7}-21 x^{5}-21 x^{4}+91 x^{3}+112 x^{2}-$ $84 x-97$ from (40). Here the particular choice of 7 th roots $\beta_{\nu}^{1 / 7}$ in (23) is determined by the automorphism $\sigma$ of $K(\zeta)$ which sends $\zeta_{49} \rightarrow \zeta_{49}^{8}$.

EXAMPLE 4. Here we wish to construct the classfield $K / k$, where $k=Q\left(\zeta_{16}+\zeta_{16}^{7}\right)$, that was described in Example 1 of the previous section. Since $K / k$ is ramified only at 5 , we seek an element $\beta$ in $k^{\prime}=Q(\sqrt{10+3 \sqrt{10}})$ for which $k\left(\zeta, \beta^{1 / 5}\right) / k(\zeta)$ is ramified only at the prime $(1-\zeta)$. 
Let $\rho$ be the element of $G\left(k^{\prime} / Q\right)$ sending $\lambda=\sqrt{10+3 \sqrt{10}}$ to $\bar{\lambda}=\sqrt{10-3 \sqrt{10}}$. Choose $\psi$ in (16) so that $\rho_{1}=1, \rho_{2}=\rho, \rho_{3}=\rho^{3}$ and $\rho_{4}=\rho^{2}$. (One can check that this choice yields the correct field $k$ here from Theorem 1 or its corollary.) Select $\varepsilon=\lambda+2$ a zero of the polynomial $q(x)=x^{4}+8 x^{3}+4 x^{2}-48 x-54$. A straightforward calculation shows that the ideal $(\lambda+2)=\mu_{1} \mu_{1}^{2} q$ as a product of primes in $k^{\prime}$, where $(3)=\rho_{1} \wedge_{2} \mu_{3} \mu_{4}$ and $(2)=q^{4}$. The primes above 3 are labelled so that $\bar{\rho}_{r}\left(\mu_{i}\right)=\mu_{i r}(\bmod 5)$, consistent with that of the $\varepsilon_{r}$ in (17). The element

$$
\beta=\beta_{1}=-148+32 \sqrt{10}+\lambda(-686+214 \sqrt{10}),
$$

obtained in (20), generates the ideal $q^{5} \rho_{1}^{5} \rho_{2}^{5} \rho_{4}^{5}$, but is not a fifth power in a completion of $k(\zeta)$ at $(1-\zeta)$. It follows from Kummer's criteria that $\beta$ may be a suitable choice. By Theorem 1, we find that $\alpha=\frac{1}{5}\left(\beta_{1}^{1 / 5}+\beta_{2}^{1 / 5}+\beta_{3}^{1 / 5}+\beta_{4}^{1 / 5}\right)$ generates some classfield of $k$ of degree 5 , and has minimal polynomial $p(x)=x^{5}+12 x^{3} / 5+$ $48 x^{2} / 25+282 x / 125+7792 / 3125$ from (39). Moreover, the Galois group $G=G(k / Q)$ satisfies (1) with $\theta(\tau)=2$. (Here $\tau$ is that generator of $V$ mapping $\zeta_{16}+\zeta_{16}^{7}$ to $\zeta_{16}^{11}+\zeta_{16}^{13}$ as in Example 1.)

It remains to verify that $k(\alpha)=K$, the desired classfield. However, a straightforward, albeit tedious, computation shows that there are only three admissible ideal groups of $k$ which have conductor divisible only by 5 , are invariant under $G(k / Q)$, and have corresponding classfields of degree 5 . They are $J_{1}=\left\{(\gamma) \mid \gamma=\alpha \cdot \beta\right.$ where $5 \nmid \alpha \beta, \alpha \in\left(k^{x}\right)^{5}$ and

$$
\beta \equiv 1+5(\delta+a(1+\sqrt{2}) \Lambda)\left(\bmod 5^{2}\right)
$$

for some integer $\delta \in Q(\sqrt{2})$ with $a \in \mathbf{Z}\}$, $J_{2}=\left\{(\gamma) \mid \gamma=\alpha \cdot \beta\right.$ where $5 \nmid \alpha \beta, \alpha \in\left(k^{x}\right)^{5}$ and

$$
\beta \equiv 1+5(a \sqrt{2}+\delta \Lambda)\left(\bmod 5^{2}\right)
$$

for some integer $\delta \in Q(\sqrt{2})$ with $a \in \mathbf{Z}\}$ and $J_{3}=J=\left\{(\gamma) \mid \gamma=\alpha \cdot \beta\right.$ where $5 \nmid \alpha \beta, \alpha \in\left(k^{x}\right)^{5}$ and

$$
\beta \equiv 1+5(\delta+a(1+2 \sqrt{2}) \Lambda)\left(\bmod 5^{2}\right)
$$

for some integer $\delta \in Q(\sqrt{2})$ with $\alpha \in \mathbf{Z}\}$.

The classfield corresponding to $J_{2}$ is seen to be $k\left(\zeta_{25}+\zeta_{25}^{7}+\zeta_{25}^{-7}+\zeta_{25}^{-1}\right)$, which is actually abelian over $Q$.

Now the norm $\eta=N_{K / k}(\Lambda-5 \alpha)=\Lambda^{5}+60 \Lambda^{2}+240 \Lambda^{2}+1410 \Lambda+$ $7792 \equiv 12-10 \sqrt{2}+\Lambda(-4+6 \sqrt{2}) \bmod 25$. Multiplying by the fifth power $\mu=[-7-7 \sqrt{2}+\Lambda(-9+6 \sqrt{2})]^{5} \equiv-7+7 \sqrt{2}+9 \Lambda \bmod 25$, 
yields $\eta \mu \equiv 1+5[1+2 \sqrt{2}-(1+2 \sqrt{2}) \Lambda] \bmod 25$. Since $(\eta)$ lies in $J$, it follows that $k(\alpha)=K$.

EXAMPLE 5. The quadratic field $k=Q(\sqrt{-47})$ has class number $h=5$, and hence admits an unramified extension $K$ of degree 5 which is normal over $Q$, with dihedral Galois group $D_{5}$. It is easy to determine that $k^{\prime}$ is generated by $\left(\zeta-\zeta^{4}\right) \sqrt{-47}=\sqrt{47 \eta \sqrt{5}}$, where $\eta=(1+\sqrt{5}) / 2$, upon choosing $\lambda=\sqrt{-47}$ in Proposition 1. To construct $K / k$ we seek, in view of Proposition 5, an element $\beta=$ $\beta_{1}$ in $k^{\prime}$ for which $k\left(\zeta, \beta^{1 / 5}\right)$ is unramified everywhere over $k(\zeta)$. Select $\varepsilon=\varepsilon_{1}=-\frac{1}{4}[47-5 \sqrt{5}+(-5+\sqrt{5}) \sqrt{47 \eta \sqrt{5}}]$, a unit in $k^{\prime}$. The element $\varepsilon$ has minimal polynomial $q(x)=x^{4}+47 x^{3}+519 x^{2}-47 x+1$ and conjugates

$$
\begin{aligned}
& \varepsilon_{2}=-\frac{1}{4}[47+5 \sqrt{5}-(5+\sqrt{5}) \sqrt{-47 \bar{\eta} \sqrt{5}}] \\
& \varepsilon_{3}=-\frac{1}{4}[47+5 \sqrt{5}+(5+\sqrt{5}) \sqrt{-47 \bar{\eta} \sqrt{5}}] \\
& \varepsilon_{4}=-\frac{1}{4}[47-5 \sqrt{5}-(-5+\sqrt{5}) \sqrt{47 \eta \sqrt{5}}]
\end{aligned}
$$

in (17). The corresponding $\beta$ from (20) and (39) are

$$
\begin{aligned}
& \beta_{1}=\frac{1}{4}[9353+4225 \sqrt{5}-(715+325 \sqrt{5}) \sqrt{47 \eta \sqrt{5}}] \\
& \beta_{2}=\frac{1}{4}[9353-4225 \sqrt{5}+(715-325 \sqrt{5}) \sqrt{-47 \bar{\eta} \sqrt{5}}] \\
& \beta_{3}=\frac{1}{4}[9353-4225 \sqrt{5}-(715-325 \sqrt{5}) \sqrt{-47 \bar{\eta} \sqrt{5}}] \\
& \beta_{4}=\frac{1}{4}[9353+4225 \sqrt{5}+(715+325 \sqrt{5}) \sqrt{47 \eta \sqrt{5}}]
\end{aligned}
$$

Since $\beta$ is a unit in $k(\zeta)$, and also a fifth power in the completion at $(1-\zeta)$, we find that $k\left(\zeta, \beta^{1 / 5}\right)$ is unramified over $k(\zeta)$. Thus the element $\alpha=\frac{1}{5}\left(\beta_{1}^{1 / 5}+\beta_{2}^{1 / 5}+\beta_{3}^{1 / 5}+\beta_{4}^{1 / 5}\right)$ generates $K / k$ and has minimal polynomial $p(x)=x^{5}+2 x^{3} / 5-47 x^{2} / 25+522 x / 125-$ $9353 / 3125$ from (39). (This is essentially the construction of $H$. Hasse [9] for the Hilbert classfield of $Q(\sqrt{-47})$.)

EXAMPLE 6. Here we wish to use the construction to find a generator $\alpha$ and its minimal polynomial for the unique cyclic field $K$, which is totally ramified at 11, unramified elsewhere, and of degree 5 over $Q$. Here $k^{\prime}=Q(\zeta)$ as in Example 5. Set $\lambda=\zeta-\zeta^{4}=\sqrt{-\sqrt{5} \eta}$ and $\lambda=$ $\zeta^{2}-\zeta^{3}=-\bar{\eta} \sqrt{-\sqrt{5} \eta}$ in $Q(\zeta)$, where $\eta=(1+\sqrt{5}) / 2$. Selecting $\psi$ in 
(16) to be the inverse of the Artin map of $Q(\zeta) / Q$ and $\varepsilon=(5+\eta \lambda) / 2$ in (17), yields $\beta=\beta_{1}=\frac{1}{44}(90-17 \sqrt{5})(50+\lambda(-5+19 \sqrt{5}))$ in (20). The element $\beta$ is a fifth power in the completion of $Q(\zeta)$ at $(1-\zeta)$, but not a global fifth power, and has norm $55^{5}$. Using Kummer's criteria, $Q\left(\zeta, \beta^{1 / 5}\right) / Q(\zeta)$ is found to be totally ramified at each prime lying above 11 , but unramified elsewhere. Hence it follows that $K$ is generated by $\alpha=\frac{1}{5}\left(\beta_{1}^{1 / 5}+\beta_{2}^{1 / 5}+\beta_{3}^{1 / 5}+\beta_{4}^{1 / 5}\right)$ with minimal polynomial $p(x)=x^{5}-3 x^{3}-3 x^{2}-x-1 / 11$ from (29). The zeros of $p(x)$ can be expressed solely in terms of real numbers. It is easy to check that the zeros are just the conjugates of $\left(\zeta_{11}+\zeta_{11}^{-1}-1\right) /\left(2-\zeta_{11}-\zeta_{11}^{-1}\right)$.

EXAMPLE 7. To illustrate the construction of classfields for which $F \neq Q$, consider the extension $k=Q(\sqrt[4]{2})$ of $F=Q(\sqrt{2})$. One finds that $k$ has narrow class number one (so does $F$ ), that $\{1+\sqrt[4]{2}, 1-\sqrt[4]{2}\}$ is a fundamental system for the units of $k$ and that $\{1, \sqrt[4]{2}, \sqrt{2}, \sqrt[4]{8}\}$ is an integral basis. The totally positive units of $k$ are generated by $1+\sqrt{2}$ and $(1+\sqrt[4]{2})^{2}$. In addition, $k / F$ is seen to correspond through classfield theory to the congruence group

$$
\begin{aligned}
\{(x) \mid x>0, x \equiv 1,3, \pm 1-\sqrt{2}, & \pm 3+\sqrt{2}, \\
3 & +2 \sqrt{2}, 1+2 \sqrt{2} \bmod 4 \sqrt{2}\}
\end{aligned}
$$

of conductor $f=(4 \sqrt{2})$. (The set $(48)$ contains precisely those ideals for which the extended Kronecker symbol $(\sqrt{2} / a)=1$.)

The field $k$ admits an ideal group $J$ defined modulo $\mathscr{M}=\left(5^{2}\right)$ consisting of principal ideals generated by elements $\gamma$ prime to $\mathscr{M}$ and of the form $\gamma=\alpha \cdot \beta$ for some $\alpha \in\left(k^{x}\right)^{5}$ and $\beta \equiv 1+$ $5(a+b \sqrt{2}+c \sqrt[4]{8}) \bmod \mathscr{M}$ with $a, b, c \in Z$. The ideal group $J$ corresponds to a classfield $K$ which is normal over $F$ with $[K: k]$ $=5$. The Galois group $G=G(K / F)$ is a semi-direct product of the form (1), say with generator $\sigma$ for $T=G(K / k)$ for which $\left[\sigma^{j}\right]=(1+5 j \sqrt{2}) J \quad(0 \leq j \leq 4)$ in (4). Also, since $\tau(\sqrt[4]{2})=-\sqrt[4]{2}$, we have $\tau\left[\sigma^{j}\right]=\left[\sigma^{-j}\right]$ so $G$ is dihedral. (A straightforward, but tedious, computation shows that $J$ is the only admissible ideal group of $k$ which has conductor divisible only by 5 , is invariant under $G(k / F)$ and has corresponding classified $K$ with $\left.G(K / F)=D_{5}.\right)$

To determine the resolvent field $k^{\prime}$, we choose $\lambda=\sqrt[4]{2}$ in Proposition 1 to find $E_{-1}^{2}=\left[\left(\zeta-\zeta^{4}\right) \sqrt[4]{2}\right]^{2}=-\eta \sqrt{10}$ where $\eta=(1+\sqrt{5}) / 2$. Hence $k^{\prime}=F(i \sqrt{\sqrt{10} \eta})$. Realizing (48) modulo $m=20 \sqrt{2}$, we find 
the sets $[\phi]$ in $(11)$ are

$$
\begin{aligned}
{\left[\phi_{1,1}\right]=\{} & (x) \mid x>0, x \equiv 1,9,11,19, \pm 1-5 \sqrt{2}, 1+10 \sqrt{2}, \\
& 3 \pm 2 \sqrt{2}, \pm 3-3 \sqrt{2}, \pm 3-7 \sqrt{2}, 3 \pm 8 \sqrt{2}, \\
& \pm 7+3 \sqrt{2}, \pm 7+7 \sqrt{2},-7 \pm 8 \sqrt{2}, 7 \pm 2 \sqrt{2}, 9+10 \sqrt{2}, \\
& \pm 9-5 \sqrt{2}, \pm 11+5 \sqrt{2}, 11+10 \sqrt{2},-13 \pm 2 \sqrt{2}, \\
& \pm 13-3 \sqrt{2}, \pm 13-7 \sqrt{2},-13 \pm 8 \sqrt{2}, \\
& 17 \pm 2 \sqrt{2}, \pm 17+3 \sqrt{2}, 17 \pm 8 \sqrt{2}, \pm 17+7 \sqrt{2}, \\
& \pm 19+5 \sqrt{2}, 19+10 \sqrt{2} \bmod 20 \sqrt{2}\}
\end{aligned}
$$

and

$$
\begin{aligned}
{\left[\phi_{\tau,-1}\right]=\{} & (x) \mid x>0, x \equiv-3,7,13,-17,-1 \pm 4 \sqrt{2}, \\
& -1 \pm 6 \sqrt{2}, \pm 1+9 \sqrt{2}, \pm 1+\sqrt{2}, \pm 3-5 \sqrt{2},-3-10 \sqrt{2}, \\
& \pm 7+5 \sqrt{2}, 7-10 \sqrt{2}, \pm 9+\sqrt{2},-9 \pm 4 \sqrt{2}, \pm 9+9 \sqrt{2}, \\
& -9 \pm 6 \sqrt{2}, \pm 11-\sqrt{2}, \pm 11+4 \sqrt{2},-11+6 \sqrt{2}, \\
& \pm 11-9 \sqrt{2}, 13-10 \sqrt{2}, \pm 13-5 \sqrt{2}, \pm 17+5 \sqrt{2}, \\
& -17-10 \sqrt{2}, \pm 19-6 \sqrt{2},-19 \pm 6 \sqrt{2},-19 \pm 4 \sqrt{2}, \\
& \pm 19-9 \sqrt{2} \bmod 20 \sqrt{2}\} .
\end{aligned}
$$

The classfield corresponding to $\mathscr{B}=\left[\phi_{1,1]} \cup\left[\phi_{\tau,-1}\right]\right.$ must be $k^{\prime}=$ $F(i \sqrt{\sqrt{10} \eta})$.

Now to construct $K / k$ we seek a suitable element $\beta$ in $k^{\prime}$ for which $k\left(\zeta, \beta^{1 / 5}\right) / k(\zeta)$ is ramified only at the prime $(1-\zeta)$ lying above 5. Let $\rho$ be the element of $G\left(k^{\prime} / F\right)$ sending $\Lambda=i \sqrt{\sqrt{10} \eta}$ to $\bar{\Lambda}=\frac{i}{\eta} \sqrt{\sqrt{10} \eta}$ and choose $\psi$ in (16) so that $\rho_{1}=1, \rho_{2}=\rho$, $\rho_{3}=\rho^{3}$ and $\rho_{4}=\rho^{2}$. (This choice of $\psi$ yields the correct field $k$ in Theorem 1 or its corollary.) Select $\varepsilon=\varepsilon_{1}=\sqrt{2}-\sqrt{5} \eta+2 \Lambda$, a unit of $k^{\prime}$ and zero of the irreducible polynomial $q(x)=x^{4}+$ $(10-4 \sqrt{2}) x^{3}+(47+10 \sqrt{2}) x^{2}+(30+2 \sqrt{2}) x+(99+70 \sqrt{2})$. Its conjugates in (17) are $\varepsilon_{2}=\sqrt{2}-\sqrt{5} \bar{\eta}+2 \Lambda / \eta, \varepsilon_{3}=\sqrt{2}-\sqrt{5} \bar{\eta}-2 \Lambda / \eta$, and $\varepsilon_{4}=\sqrt{2}-\sqrt{5} \eta-2 \Lambda$. The corresponding $\beta=\beta_{1}$, from (20) and (39) is

$$
\begin{aligned}
& \frac{1}{2}((-89325+64303 \sqrt{2})+\sqrt{5}(40007-28715 \sqrt{2})) \\
& \quad+\Lambda((32737-22440 \sqrt{2})+\sqrt{5}(-14651+10028 \sqrt{2})) .
\end{aligned}
$$

Since $\beta$ is not a fifth power in the completion of $k(\zeta)$ at $(1-\zeta)$, we find from Theorem 1 that $\alpha=\frac{1}{5}\left(\beta_{1}^{1 / 5}+\beta_{2}^{1 / 5}+\beta_{3}^{1 / 5}+\beta_{4}^{1 / 5}\right)$ generates 
some classfield of $k$ of degree 5. This classfield must be $K$ in view of the parenthetical remark made about $J$. From (39), $\alpha$ has minimal polynomial

$$
\begin{aligned}
p(x)= & x^{5}-(19+10 \sqrt{2}) / 5 x^{3}+(30+2 \sqrt{2}) / 25 x^{2} \\
& +(38+20 \sqrt{2}) / 125 x+(179900-127256 \sqrt{2}) / 3125 .
\end{aligned}
$$

EXAMPLE 8. In this final illustration, we wish to apply the construction to find a generator $\alpha$ and minimal polynomial for the real subfield $K$ of $Q\left(\zeta_{17}\right)$. Again $k^{\prime}=Q(\zeta)$ since $k=Q$. Selecting $\varepsilon_{r}=2+\zeta^{r}$ in $(41)$ yields

$$
\begin{aligned}
\beta=\beta_{1} & =(4+i)^{2}(3+2 i \sqrt{2})^{4}(4+i+2 \sqrt{2}+2 i \sqrt{2})^{4} / 17^{3}, \\
\beta_{2} & =(4-i)^{2}(3+2 i \sqrt{2})^{4}(4-i-2 \sqrt{2}+2 i \sqrt{2})^{4} / 17^{3}, \\
\beta_{3} & =(4+i)^{2}(3-2 i \sqrt{2})^{4}(4+i-2 \sqrt{2}-2 i \sqrt{2})^{4} / 17^{3}, \\
\beta_{4} & =(4-i)^{2}(3-2 i \sqrt{2})^{4}(4-i+2 \sqrt{2}-2 i \sqrt{2})^{4} / 17^{3}
\end{aligned}
$$

in (20). Now it follows from Proposition 5 that $Q\left(\zeta, \beta^{1 / 8}\right) / Q(\zeta)$ is totally ramified at each $Q(\zeta)$-prime lying above 17 , and unramified at other primes except possibly at $(1+\zeta)$ lying above 2 . Hcwever, it can be checked that $\beta$ is an eighth power in the completion of $Q(\zeta)$ at $(1+\zeta)$. For any $Q(\zeta)$-prime $\rho$ lying above 17 , we have $e(\tilde{\mu}: \mu)$ $=8$, so (46) holds. As $K$ is the unique cyclic field with such ramification, it is generated by $\alpha=\frac{1}{8}\left(\beta_{1}^{1 / 8}+\beta_{3}^{1 / 8}+\beta_{5}^{1 / 8}+\beta_{7}^{1 / 8}\right)$ for our construction. The minimal polynomial for $\alpha$ is $p(x)=x^{8}-20 x^{6} /\left(2^{4}\right)$ $+1986 x^{4} /\left(2^{8} \cdot 17\right)-50256 x^{2} /\left(2^{12} \cdot 17^{2}\right)+300304 /\left(2^{16} \cdot 17^{3}\right)$ from $(41)$, and has zeros

$$
\pm \frac{1}{4 \sqrt{17}} \sqrt{(85 \pm \sqrt{17}) \mp \sqrt{4777+409 \sqrt{17}}}
$$

expressed solely in terms of real numbers.

\section{REFERENCES}

[1] E. Artin and J. Tate, Classfield Theory, Benjamin, New York, 1968.

[2] Z. I. Borevich and I. R. Shafarevich, Number Theory, Academic Press, New York, 1966.

[3] L. E. Dickson, Elementary Theory of Equations, Wiley, New York.

[4] S. Gurak, Ideal-theoretic characterization of the relative genus field, J. Reine Angew. Math., 296 (1977), 119-125.

[5] _ On the representation theory for full decomposable forms, J. Number Theory, 13, no. 4 (1981), 421-442. 
[6] Cubic and Biquadratic Pseudoprimes of Lucas Type, in Number Theory (edited by J. M. De Koninck \& C. Levesque), Walter de Gruyter, Berlin, 1989.

[7] _ Minimal polynomials for circular numbers, Pacific J. Math., 112, no. 2 (1984), 313-331.

[8] _ Pseudoprimes for higher order linear recurrence sequences, Math. Comp., 55, no. 192 (1990), 783-813.

[9] H. Hasse, Uber den Klassenkorper zum quadratischen Zahlkorper mit diskriminante -47, Acta. Arith., 9 (1964), 419-434.

[10] S. Iyanaga, Class-Field Theory Notes, Univ. of Chicago, 1961.

[11] G. Janusz, Algebraic Number Fields, Academic Press, New York, 1973.

[12] J. Rotman, The Theory of Groups, Allyn \& Bacon, Boston, 1970.

[13] B. Setzer, The determination of all imaginary quartic Abelian number fields with class number 1, Math. Comp., 35, no. 152 (1980), 1383-1386.

[14] B. L. van der Waerden, Modern Algebra, vol. I, Unger, New York, 1949.

Received August 5, 1991 and in revised form October 1, 1991.

UNIVERSITY OF SAN Diego

SAN Diego, CA 92110-2492 


\title{
PACIFIC JOURNAL OF MATHEMATICS
}

\author{
Founded by
}

E. F. BECKENBACH (1906-1982) F. WoLF (1904-1989)

\section{EDITORS}

$\begin{array}{ll}\begin{array}{l}\text { V. S. VARADARAJAN } \\ \text { (Managing Editor) }\end{array} & \begin{array}{l}\text { NiCHOLAS ERCOLANI } \\ \text { University of Arizona }\end{array} \\ \text { University of California } & \text { Tucson, AZ 85721 } \\ \text { Los Angeles, CA 90024-1555 } & \text { ercolani@math.arizona.edu } \\ \text { vsv@math.ucla.edu } & \text { R. FinN } \\ \text { HERBERT CLEMENS } & \text { Stanford University } \\ \text { University of Utah } & \text { Stanford, CA 94305 } \\ \text { Salt Lake City, UT 84112 } & \text { finn@gauss.stanford.edu } \\ \text { clemens@math.utah.edu } & \text { VAUGHAN F. R. JoNEs } \\ \text { F. MichAEL CHRIsT } & \text { University of California } \\ \text { University of California } & \text { Berkeley, CA 94720 } \\ \text { Los Angeles, CA 90024-1555 } & \text { vfr@math.berkeley.edu } \\ \text { christ@math.ucla.edu } & \text { STEVEN KERCKHoFF } \\ \text { THomas ENRIGHT } & \text { Stanford University } \\ \text { University of California, San Diego } & \text { Stanford, CA 94305 } \\ \text { La Jolla, CA 92093 } & \text { spk@gauss.stanford.edu } \\ \text { tenright@ucsd.edu } & \end{array}$

\author{
C. C. MOORE \\ University of California \\ Berkeley, CA 94720
}

MaRTin ScharlemanN

University of California

Santa Barbara, CA 93106

mgscharl@henri.ucsb.edu

\author{
HAROLD STARK \\ University of California, San Diego \\ La Jolla, CA 92093
}

\begin{tabular}{ll}
\multicolumn{1}{c}{ SUPPORTING } & INSTITUTIONS \\
UNIVERSITY OF ARIZONA & UNIVERSITY OF OREGON \\
UNIVERSITY OF BRITISH COLUMBIA & UNIVERSITY OF SOUTHERN CALIFORNIA \\
CALIFORNIA INSTITUTE OF TECHNOLOGY & STANFORD UNIVERSITY \\
UNIVERSITY OF CALIFORNIA & UNIVERSITY OF HAWAII \\
MONTANA STATE UNIVERSITY & UNIVERSITY OF TOKYO \\
UNIVERSITY OF NEVADA, RENO & UNIVERSITY OF UTAH \\
NEW MEXICO STATE UNIVERSITY & WASHINGTON STATE UNIVERSITY \\
OREGON STATE UNIVERSITY & UNIVERSITY OF WASHINGTON
\end{tabular}

The Supporting Institutions listed above contribute to the cost of publication of this Journal, but they are not owners or publishers and have no responsibility for its content or policies.

Mathematical papers intended for publication in the Pacific Journal of Mathematics should be in typed form or offset-reproduced (not dittoed), double spaced with large margins. Please do not use built up fractions in the text of the manuscript. However, you may use them in the displayed equations. Underline Greek letters in red, German in green, and script in blue. The first paragraph must be capable of being used separately as a synopsis of the entire paper. In particular it should contain no bibliographic references. Please propose a heading for the odd numbered pages of less than 35 characters. Manuscripts, in triplicate, may be sent to any one of the editors. Please classify according to the 1991 Mathematics Subject Classification scheme which can be found in the December index volumes of Mathematical Reviews. Supply name and address of author to whom proofs should be sent. All other communications should be addressed to the managing editor, or Julie Speckart, University of California, Los Angeles, California 90024-1555.

There are page-charges associated with articles appearing in the Pacific Journal of Mathematics. These charges are expected to be paid by the author's University, Government Agency or Company. If the author or authors do not have access to such Institutional support these charges are waived. Single authors will receive 50 free reprints; joint authors will receive a total of 100 free reprints. Additional copies may be obtained at cost in multiples of 50 .

The Pacific Journal of Mathematics (ISSN 0030-8730) is published monthly except for July and August. Regular subscription rate: $\$ 190.00$ a year (10 issues). Special rate: $\$ 95.00$ a year to individual members of supporting institutions.

Subscriptions, orders for numbers issued in the last three calendar years, and changes of address should be sent to Pacific Journal of Mathematics, P.O. Box 969, Carmel Valley, CA 93924, U.S.A. Old back numbers obtainable from Kraus Periodicals Co., Route 100, Millwood, NY 10546.

The Pacific Journal of Mathematics at P.O. Box 969, Carmel Valley, CA 93924 (ISSN 0030-8730) is published monthly except for July and August. Second-class postage paid at Carmel Valley, California 93924, and additional mailing offices. Postmaster: send address changes to Pacific Journal of Mathematics, P.O. Box 969, Carmel Valley, CA 93924.

PUBLISHED BY PACIFIC JOURNAL OF MATHEMATICS, A NON-PROFIT CORPORATION Copyright $(\mathcal{1} 1993$ by Pacific Journal of Mathematics 


\section{PACIFIC JOURNAL OF MATHEMATICS}

Volume $157 \quad$ No. $2 \quad$ February 1993

Strong integral summability and the Stone-Čech compactification of the half-line

JEFF CONNOR and MARY ANNE SWARDSON

The endlich Baer splitting property

225

THEODORE GERARD FATICONI

The formal group of the Jacobian of an algebraic curve

MARGARET N. FREIJE

Concordances of metrics of positive scalar curvature

PAWEL GAJER

Explicit construction of certain split extensions of number fields and constructing cyclic classfields

STANLEY JOSEPH GURAK

Asymptotically free families of random unitaries in symmetric groups

ALEXANDRU MiHAi NiCA

On purifiable subgroups and the intersection problem

TAKASHI OKUYAMA

On the incidence cycles of a curve: some geometric interpretations

LUCIANA RAMELLA

On some explicit formulas in the theory of Weil representation

R. RANGA RAO

An analytic family of uniformly bounded representations of a free product of 373 discrete groups

JANUSZ WYSOCZAŃSKI

Errata: "Dentability, trees, and Dunford-Pettis operators on $L_{1}$ "

MARIA GIRARDI and ZHIBAO HU

Errata: "Poincaré cobordism exact sequences and characterisation" 